\title{
Compositional Variability of Monazite-Cheralite-Huttonite Solid Solutions, Xenotime, and Uraninite in Geochemically Distinct Granites with Special Emphasis to the Strongly Fractionated Peraluminous Li-F-P-Rich Podlesí Granite System (Erzgebirge/Krušné Hory Mts., Central Europe)
}

\author{
Karel Breiter ${ }^{1, *(\mathbb{D})}$ and Hans-Jürgen Förster ${ }^{2}$ (D) \\ Czech Academy of Sciences, Institute of Geology, Rozvojová 269, Praha 6, CZ-16500 Prague, Czech Republic \\ 2 Helmholtz Centre Potsdam, GFZ German Research Centre for Geosciences, 14473 Potsdam, Germany; \\ forhj@gfz-potsdam.de \\ * Correspondence: breiter@gli.cas.cz
}

Citation: Breiter, K.; Förster, H.-J. Compositional Variability of Monazite-Cheralite-Huttonite Solid Solutions, Xenotime, and Uraninite in Geochemically Distinct Granites with Special Emphasis to the Strongly Fractionated Peraluminous Li-F-P-Rich Podlesí Granite System (Erzgebirge/Krušné Hory Mts., Central Europe). Minerals 2021, 11, 127. https://doi.org10.3390/ $\min 11020127$

Academic Editor: Jarda Dostal Received: 8 December 2020

Accepted: 21 January 2021

Published: 27 January 202

Publisher's Note: MDPI stays neutral with regard to jurisdictional claims in published maps and institutional affiliations.

Copyright: (c) 2021 by the authors. Licensee MDPI, Basel, Switzerland. This article is an open access article distributed under the terms and conditions of the Creative Commons Attribution (CC BY) license (https:// creativecommons.org/licenses/by/ $4.0 /)$

\begin{abstract}
A comprehensive study of monazite-cheralite-huttonite solid solutions (s.s.) and xenotime from the highly evolved, strongly peraluminous P-F-Li-rich Podlesí granite stock in the Krušné Hory Mts., Czech Republic, indicates that, with the increasing degree of magmatic and high-T early post-magmatic evolution, the content of the cheralite component in monazite increases and the relative dominance of middle rare earth elements (MREE) in xenotime becomes larger. Considering the overall compositional signatures of these two accessory minerals in the late Variscan granites of the Erzgebirge/Krušné Hory Mts., three types of granites can be distinguished: (i) chemically less evolved F-poor S(I)- and A-type granites contain monazite with a smooth, mostly symmetric chondrite-normalized $(\mathrm{CN})$ rare-earth elements (REE) pattern gradually declining from La to Gd; associated xenotime is Y-rich ( $>0.8$ apfu Y) with a flat MREE-HREE (heavy rare earth elements) pattern; (ii) fractionated A-type granites typically contain La-depleted monazite with Th accommodated as the huttonite component, combined with usually Y-poor (0.4-0.6 apfu Y) xenotime characterized by a smoothly inclining, Yb-Lu-dominant CN-REE pattern; (iii) fractionated peraluminous Li-mica granites host monazite with a flat, asymmetric (kinked at La and Nd) CN-LREE pattern, with associated xenotime distinctly MREE (Gd-Tb-Dy)-dominant. Monazite and xenotime account for the bulk of the REE budgets in all types of granite. In peraluminous S(I)-type granites, which do not bear thorite, almost all Th is accommodated in monazite-cheralite s.s. In contrast, Th budgets in A-type granites are accounted for by monazite-huttonite s.s. together with thorite. The largest portion of $U$ is accommodated in uraninite, if present.
\end{abstract}

Keywords: monazite; cheralite; xenotime; uraninite; Erzgebirge/Krušné Hory

\section{Introduction}

In granitic rocks, radioactive elements ( $U$, Th) and rare-earth elements (REE) plus $Y$, together with other high-field-strength elements (HFSE) like $\mathrm{Zr}, \mathrm{Nb}$ and $\mathrm{Ta}$, are predominantly hosted in accessory minerals, namely monazite, xenotime, apatite, zircon, allanite, and uraninite. Bea [1] calculated that accessory minerals accounted for $75-90 \%$ of the budget of these elements in peraluminous granites. Förster [2,3] performed mass-balance calculations for REE Th and $U$ and established that monazite, xenotime, and uraninite typically contain between 80 and $95 \%$ of the elemental budgets in Variscan granites of the German Erzgebirge. Trumbull et al. [4] estimated the proportion of monazite and xenotime in the Th and REE budgets of the A-type Erongo granite at approximately 85-95\%. Breiter [5] calculated that 78-95\% of Th in peraluminous granites of the Moldanubian Zone of the Variscan orogen in the Czech Republic are hosted by monazite. All these results 
stressed the significance of accessory phases in the evolution of the U, Th, Y, and REE budgets during magmatic fractionation and the subsequent late magmatic to post-magmatic hydrothermal overprinting in granitic rocks.

During our long-term systematic petrological study of granites in the Erzgebirge/ Krušné Hory area (Germany, Czech Republic), we collected several thousands of microprobe spot analyses of monazite, xenotime, and uraninite. Some of them had already been published [2,3,5-10], but a large part still remained unreported, especially data related to the unique, extremely evolved Podlesí P-F-Li-rich granite system. We decided to present a review of the entire set of accessory mineral data we have assembled in the past years from the diverse Erzgebirge/Krušné Hory granites with three major goals: (i) assessment of the influence of magmatic and hydrothermal processes on the composition of monazite, xenotime, and uraninite within an extremely fractionated, fluid- and volatile-rich granite system (Podlesí case study); (ii) capturing the overall diversity of monazite and xenotime compositions in different types of ore-bearing and barren granites of the Erzgebirge/Krušné Hory; and (iii) an attempt to establish basic relations between monazite and xenotime compositions and the bulk-rock composition of the parental granite.

\section{Geology}

\subsection{Variscan Granites of the Erzgebirge/Krušné Hory Area}

Variscan granites in the Erzgebirge/Krušné Hory, together with those emplaced in the adjacent Fichtelgebirge and Slavkovský Les areas, represent one of the most comprehensively studied and long-term-studied suites of ore-bearing granites worldwide [11-14].

The detailed study of these granites goes back to the middle of the 19th century, when the ore-bearing and barren granite varieties were first distinguished [15]. Later, Teuscher [16] first suggested a pervasive post-magmatic metasomatic overprint being crucial in the evolution of tin-bearing granites. From the various classification schemes elaborated for the Erzgebirge/Krušné Hory granites during the last decades [11,12,14,17], in this study, we use the classification proposed by Förster [12] based on typical mineral/geochemical features of individual plutons (Figure 1):

1. Low-F peraluminious biotite granites are abundant throughout the whole Erzgebirge (i.e., the Kirchberg pluton and a part of the Nejdek pluton in the west, Lesná granite in the central Erzgebirge, and the Fláje, Niederbobritsch, and Telnice plutons in the east);

2. Low-F two-mica peraluminous granites represented by the Bergen massif in the western Erzgebirge; similar granites are more common in the adjacent Fichtelgebirge and Slavkovský Les areas;

3. P-F-Li-rich peraluminous Li-mica granites form large outcropping bodies in the west (the Eibenstock-Nejdek pluton) and several, mostly hidden, bodies in the central Erzgebirge (Geyer, Ehrenfriedersdorf, Annaberg, Satzung). Micas in these granites evolved from Li-enriched biotite in less fractionated granites to zinnwaldite in laterformed, evolved granites forming small stocks; topaz is common;

4. F-Li-rich and P-poor granites of A-type chemical signature which form less voluminous plutons, comprising the occurrences of strongly mineralized granite in the eastern Erzgebirge (Schellerhau, Sadisdorf, Altenberg, Zinnwald/Cínovec and Krupka). Micas in these granites are well evolved from Li-enriched biotite to zinnwaldite; magmatic fluorite is common;

5. Medium-F and P-poor biotite-to-Li-mica granites of A-type chemical signature form dykes, stocks, and breccia pipes in the central (Seifen, Hora svaté Kateřiny) and western (the Gottesberg-Schneckenstein dyke swarm) parts of the province. 


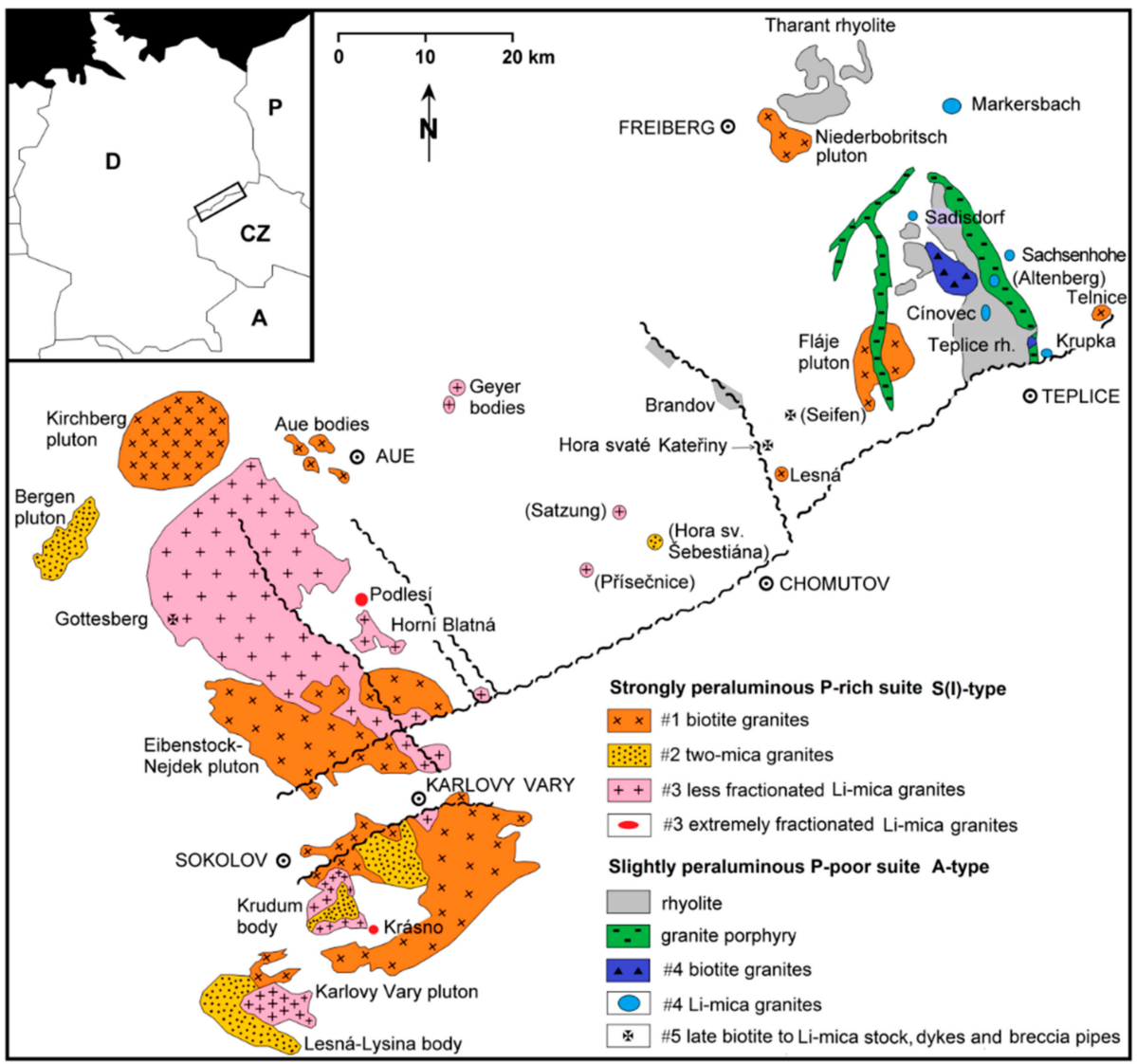

Figure 1. A sketch of the distribution of late Variscan granites in the Erzgebirge/Krušné Hory and adjacent Slavkovský Les area. Classification of granites into five geochemical groups according to [12]. Names of hidden bodies known only from boreholes or mines are given in parentheses.

According to well-documented intrusive contacts, group \#3 is younger than group \#1, and group \#5 is younger than group \#3. Intrusive ages of groups 1-3 lie most probably in the interval of 327-315 Ma [13,18], while group \#5 is substantially younger: 305-295 Ma [9,19]. The age of group \#4 is still highly controversial, lying within the time interval of 325$310 \mathrm{Ma}$, according to $[20,21]$ and references therein. Group 1-3 granites are transitional late-collisional S-I-type rocks, with the proportion of metasedimentary material being larger in F-Li-P-rich granites and comparatively smaller in the two other groups. Granites of groups $4-5$ belong to the class of aluminous A-type rocks emplaced in a late collisional to post-collisional setting.

Mineralogical, geochemical, isotopic, and geochronological data on the herein-discussed Erzgebirge/Krušné Hory granites were the subject of numerous papers (for overview, see $[3,13,14,17])$ and are not reported here in detail. Because only a limited number of mineral data from the group \#2 granites were available, we merged groups \#1 and \#2 into a group of peraluminous biotite and two-mica granites in the discussion. Similarly, due to the uncertain age of some intrusions, we merged the geochemically similar groups \#4 and \#5 into a group of A-type granites.

\subsection{The Strongly Fractionated Podlesi Granite System}

The Podlesí granite system (outcrop $0.1 \mathrm{~km}^{2}$ ) is situated in the western part of the Krušné Hory/Erzgebirge Mts. It is the relatively youngest intrusion within the multistage Eibenstock-Nejdek pluton, representing the most fractioned body of the peraluminous Li-mica granite group \#3 (Figure 2) [22,23]. It is neighbored by Li-biotite granite of the Nejdek pluton, also belonging to the peraluminous Li-mica granite group [12]. 
Table 1. The studied samples from Podlesí.

\begin{tabular}{|c|c|c|c|}
\hline $\begin{array}{c}\text { Assemblage, } \\
\text { Compare Figure } 2\end{array}$ & Sample No. & Sample Location and Description & $\begin{array}{c}\text { Analyzed Minerals and Number } \\
\text { of Analyses }\end{array}$ \\
\hline A & 3361 & $\begin{array}{l}\text { stockscheider (marginal pegmatite) at the } \\
\text { uppermost contact of the stock, thickness of the } \\
\text { stockscheider layer is } 20-40 \mathrm{~cm} \text {, composed of red } \\
\text { Kfs crystals up to } 5 \times 2 \mathrm{~cm} \text { in size and } \\
\text { fine-grained Qtz-Ab-protolithionite matrix }\end{array}$ & Mnz (10), Xnt (9) \\
\hline B & 3385 & $\begin{array}{c}\text { Protolithionite granite, outcrop in the upper part } \\
\text { of the stock, fine-grained granite composed of } \\
\text { Qtz, Kfs, Ab, protolithionite, and Toz }\end{array}$ & $\operatorname{Mnz}(10)$, Xnt (3) \\
\hline $\mathrm{C}$ & 3365 & $\begin{array}{c}\text { mica-rich greisen in the upper part of the stock, } \\
\text { boulders, dark greisen composed of F- and } \\
\text { Fe-rich Li-poor mica and Qtz (+ Toz +Ap), } \\
\text { feldspars only in relicts }\end{array}$ & Mnz (19), Ur (2) \\
\hline $\mathrm{D}$ & 3389 & $\begin{array}{c}\text { quartz-rich greisen in the upper part of the stock, } \\
\text { outcrop, a fully greisenized flat dyke of } \\
\text { zinnwaldite granite composed of Qtz (+ } \\
\text { Zinnwaldite +Toz + Wolframite) }\end{array}$ & $\operatorname{Mnz}(1), \operatorname{Ur}(1)$ \\
\hline $\mathrm{E}$ & 3413 & $\begin{array}{l}\text { zinnwaldite granite, quarry, lower part of the } \\
\text { main flat dyke with normal medium-grained } \\
\text { granitic texture, composed of Qtz, P-rich Kfs, } \\
\text { P-rich Ab, Zinnwaldite, and Toz }\end{array}$ & Cher (5) \\
\hline $\mathrm{F}$ & 3436,3443 & $\begin{array}{c}\text { protolithionite granite, borehole PTP-1, depths } \\
200 \text { and } 290 \mathrm{~m} \text {, medium-grained granite } \\
\text { composed of Qtz, Kfs, Ab, protolithionite, and } \\
\text { Toz }\end{array}$ & $\operatorname{Mnz}(16)$, Xnt (4), Ur (13) \\
\hline G & 4206,4208 & $\begin{array}{l}\text { Li-biotite granite, borehole PTP-5, depths } 114 \\
\text { and } 136 \mathrm{~m} \text {, composed of Qtz, Kfs, Ab, Bt (+ Toz) }\end{array}$ & $\operatorname{Mnz}(12)$, Xnt (6) \\
\hline
\end{tabular}

Mineral abbreviations according to [24].

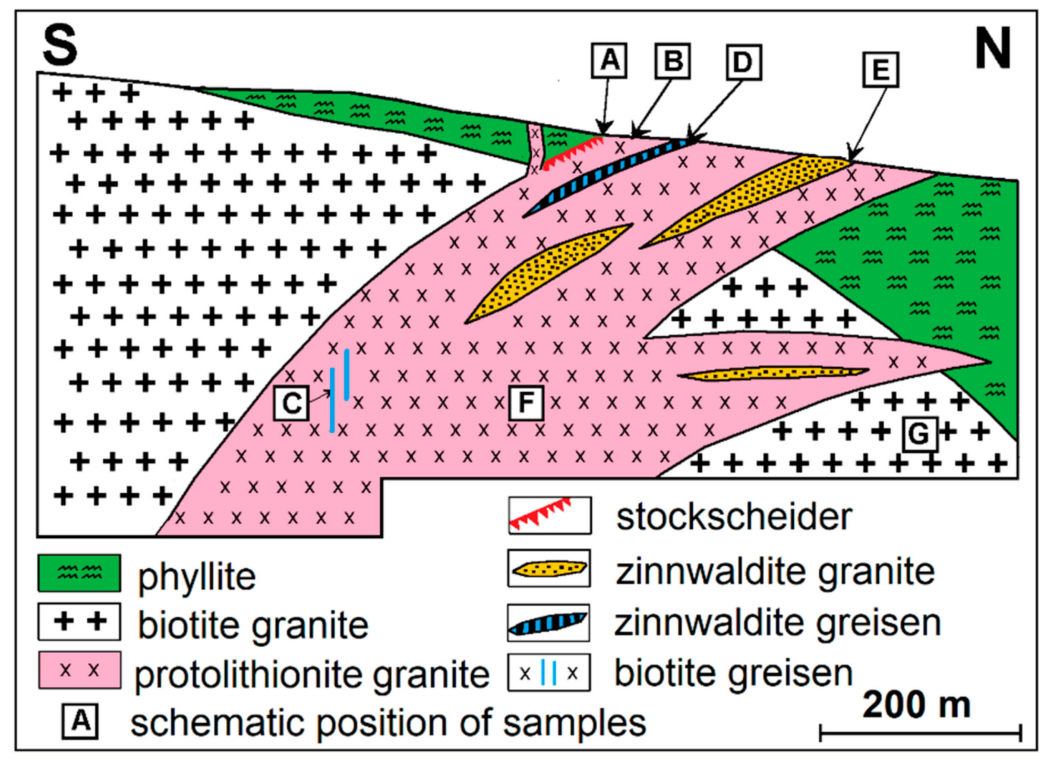

Figure 2. A simplified cross-section through the Podlesí granite stock with positions of samples. For explanation of symbols A-G see the Table 1 .

The overall shape of the intrusion is tongue-like. Its upper contact is bordered by a $50 \mathrm{~cm}$ thick layer of stockscheider, i.e., a zone composed of large, prismatic microcline crystals oriented perpendicular to the contact plane of the granite with phyllite. The body is 
generally composed of albite-protolithionite-topaz granite, which can be divided into two sub-facies: The "upper facies" builds the uppermost 30-40 m of the stock and is fine-grained and porphyritic. The "lower facies", forming the main part of the stock, is medium-grained and non-porphyritic. In the uppermost $100 \mathrm{~m}$, the stock granite is intruded by several generally flat-lying dykes of albite-zinnwaldite-topaz granite with a maximum thickness of $7 \mathrm{~m}$. Upper and lower contacts of the dykes are sharp and relatively flat.

The Podlesí magmatic system was explosively opened two times. The first episode of opening postdated the crystallization of the stockscheider. The resultant explosive breccia is composed of angular fragments of phyllite and stockscheider cemented by finegrained granitic matrix. The second episode of opening succeeded the emplacement of the flat dykes: fragments of mica-rich dyke facies 1-10 cm across were cemented by the latest portion of mica-poor residual melt. After this opening, prominent manifestation of magmatic layering and unidirectional solidification textures (UST) evolved. The most significant UST layer is defined by orthoclase, locally also by quartz + zinnwaldite [23].

Post-magmatic hydrothermal processes (greisenization) are confined to rare, thin, and steep stringers of biotite-rich greisen in the protolithionite granite and local quartz-rich greisenization developed in the uppermost dyke of the zinnwaldite granite [23].

Geochemically, the protolithionite granite is strongly peraluminous (A/CNK 1.15$1.25)$ and, compared to common peraluminous granites, rich in phosphorus $(0.4-0.8 \mathrm{wt} . \%$ $\mathrm{P}_{2} \mathrm{O}_{5}$ ), fluorine (0.6-1.8 wt.\% F), and incompatible elements, such as $\mathrm{Li}, \mathrm{Rb}, \mathrm{Cs}, \mathrm{Sn}, \mathrm{Nb}, \mathrm{W}$, and $\mathrm{U}$, and poor in $\mathrm{V}, \mathrm{Sc}, \mathrm{Ni}, \mathrm{Co}, \mathrm{Mg}, \mathrm{Ca}, \mathrm{Sr}, \mathrm{Ba}, \mathrm{Fe}, \mathrm{Zr}, \mathrm{REE}, \mathrm{Th}$, and $\mathrm{Pb}$. The high degree of magmatic fractionation is demonstrated by low $\mathrm{K} / \mathrm{Rb}$ and $\mathrm{Zr} / \mathrm{Hf}$ values (22-35 and $12-20$, respectively). The zinnwaldite granite is even richer in $\mathrm{Al}(\mathrm{A} / \mathrm{CNK}=1.2-1.4), \mathrm{P}$ (0.6-1.5 wt.\% $\left.\mathrm{P}_{2} \mathrm{O}_{5}\right), \mathrm{F}(1.4-2.4$ wt.\%), Na, Rb, Li, Nb, and Ta and poor in $\mathrm{Si}, \mathrm{Zr}, \mathrm{Sn}, \mathrm{W}$, and $\mathrm{REE}$. The $\mathrm{K} / \mathrm{Rb}(14-20)$ and $\mathrm{Zr} / \mathrm{Hf}(9-13)$ ratios are lower than those of the protolithionite granite. The contents of Th, Y, and REEs in the protolithionite and zinnwaldite granite are comparatively low: 4-8 and 4-6 ppm Th, respectively; 4-10 and 1.5-6 ppm Y, respectively; and 10-25 and 3-10 ppm total REE, respectively. The concentrations of $U$ are large and approach 25-40 and 20-25 ppm, respectively.

\section{Methods}

Minerals in polished thin sections were analyzed using a Camebax SX 50 microprobe at GFZ Potsdam, and a Cameca SX100 microprobe housed at Masaryk University Brno. The operating conditions in Brno involved an accelerating voltage of $15 \mathrm{kV}$, a beam current of $20 \mathrm{nA}$, and a beam diameter of $2 \mu \mathrm{m}$. The following standards, X-ray lines, and crystals (in parentheses) were used: $\mathrm{Si} K_{\alpha}$-sanidine (TAP); $\mathrm{P} K_{\alpha}, \mathrm{CaK}_{\alpha}$-fluorapatite (PET); $\mathrm{YL}_{\alpha}-\mathrm{YPO}_{4}$ (PET); $\mathrm{LaL}_{\alpha}-\mathrm{LaPO}_{4}(\mathrm{PET}) ; \mathrm{CeL}_{\alpha}-\mathrm{CePO}_{4}(\mathrm{PET}) ; \mathrm{Dy}_{\alpha}-\mathrm{DyPO}_{4}(\mathrm{LIF}) ; \mathrm{ErL}_{\alpha}-\mathrm{ErPO}_{4}(\mathrm{PET})$; $\mathrm{Pr}_{\beta}-\mathrm{PrPO}_{4}$ (LIF); $\mathrm{Nd}_{\beta}-\mathrm{NdPO}_{4}$ (LIF); $\mathrm{SmL}{ }_{\beta}-\mathrm{SmPO}_{4}$ (LIF); $\mathrm{Nd} L_{\beta}-\mathrm{NdPO}_{4}(\mathrm{LIF}) ; \mathrm{Gd}_{\beta^{-}}$ $\mathrm{GdPO}_{4}(\mathrm{LIF}) ; \mathrm{ThM} \mathrm{\alpha}_{\alpha}-\mathrm{CaTh}\left(\mathrm{PO}_{4}\right)_{2}(\mathrm{PET}) ; \mathrm{PbM} \mu_{\alpha}$-vanadinite (PET); and $\mathrm{UM}_{\beta}$-metallic U (PET). Intra-REE overlaps were partially resolved by using $L_{\alpha}$ and $L_{\beta}$ lines. Empirically determined correction equations for peak overlaps were applied.

The operating conditions in Potsdam were as follows: accelerating potential was $20 \mathrm{kV}$; beam current was 40-60 nA; beam diameter was 1-2 $\mu \mathrm{m}$; counting times on the peak (background) were $300 \mathrm{~s}$ (150 s) for Pb, $200 \mathrm{~s}$ (100 s) for U and Th, and $60 \mathrm{~s} \mathrm{(40} \mathrm{s)} \mathrm{for} \mathrm{other}$ elements. The following analyzing crystals were used: LIF for REE and Fe; TAP for Si, Al, and Y; and PET for P, Th, U, Ca, and Pb. For more details, see [5-7].

The contents of some elements, typically Ho in monazite and La and Pr in xenotime, are at their limits of detection. After a careful evaluation, we decided to report all values exceeding their calculated limits of detection to the tables and figures, but remained as conservative as possible in their interpretation.

Structural formulae of monazite-cheralite-huttonite s.s. and xenotime were calculated on the basis of 4 atoms of oxygen in a formula unit ( $4 \mathrm{O} a p f u$ ), those of uraninite on the basis of 2 atoms of oxygen in a formula unit ( $2 \mathrm{O}$ apfu). 
The whole dataset considered in this study encompasses 252 analyses of monazite, incl. monazite-cheralite and monazite-huttonite solid solutions, 113 analyses of xenotime, and 34 analyses of uraninite.

Terminological remarks:

1. The minerals designated as "monazite" or "xenotime" in this paper are, according to the IMA-CNMMN nomenclature, monazite-(Ce) and xenotime-(Y). We used abbreviated names in order to make the text more fluent.

2. According to IMA-CNMNM rules, only endmember names should be used in the mica group of minerals; i.e., all Li-Fe mica varieties should be described either as annite/siderophyllite or as lepidolite. This is, from the petrological point of view, unsatisfactory. Similarly, as in the case of feldspars, a more elaborated classification is demand. Here, we used the traditional names protolithionite and zinnwaldite for micas with ideal compositions, namely $\mathrm{K}_{2} \mathrm{LiFe}_{4} \mathrm{Al}\left(\mathrm{Al}_{2} \mathrm{Si}_{6} \mathrm{O}_{20}\right)(\mathrm{F}, \mathrm{OH})_{4}$ and $\mathrm{K}_{2} \mathrm{Li}_{2} \mathrm{Fe}_{2} \mathrm{Al}_{2}\left(\mathrm{Al}_{2} \mathrm{Si}_{6} \mathrm{O}_{20}\right) \mathrm{F}_{4}$, respectively.

\section{Results}

Monazite, xenotime and uraninite were studied in representative samples from the entire Podlesí granite system (Figure 2). A list of samples and their bulk-rock compositions are provided in Table 1 and as Supplementary Electronic Material S1. Typical shapes of monazite, xenotime, and uraninite crystals and their intergrowths are presented in Figure 3. The results of representative spot analyses are listed in Tables 2-4, respectively. The entire set of yet unpublished analyses (111 spots) is provided as Supplementary Electronic Material S2.

\subsection{Monazite-Cheralite-Huttonite Solid Solutions}

Monazite is common at Podlesí in all varieties of the protolithionite granite as well as in the surrounding Li-biotite granite and the greisens. In contrast, monazite sensu stricto is absent from the dykes of zinnwaldite granite, where instead its Th-dominated variety, cheralite (ideally CaTh $\left.\left(\mathrm{PO}_{4}\right)_{2}\right)$, crystallized. Monazite usually forms homogeneous, isometric to columnar grains hosted in mica flakes (Figure 3a,b). Zoning patterns, with Th contents increasing towards the rims of the grains, were rarely observed in backscattered electron (BSE) images (Figure 3c), while monazite closely associated with zircon and xenotime in the cores of mica flakes is common (Figure 3d,e). The composition of monazite from the protolithionite granite incl. stockscheider does not vary significantly, being dominated by light rare earth elements (LREE): 15-22 wt $\% \mathrm{Ce}_{2} \mathrm{O}_{3}, 7.2-10.5 \mathrm{wt} \%$ $\mathrm{Nd}_{2} \mathrm{O}_{3}, 4.4-8.2 w^{\circ} \% \mathrm{La}_{2} \mathrm{O}_{3}, 1.9-2.6 \mathrm{wt} \% \mathrm{Pr}_{2} \mathrm{O}_{3}, 1.5-2.8 w t \% \mathrm{Sm}_{2} \mathrm{O}_{3}$, and $\mathrm{Y}\left(2.4-4.5 w \mathrm{t}^{\%}\right.$ $\mathrm{Y}_{2} \mathrm{O}_{3}$ ) (Table 2).

Contents of middle rare earth elements (MREE) and heavy rare earth elements (HREE) are substantially lower: $<0.1-3.4 \mathrm{wt} \% \mathrm{Gd}_{2} \mathrm{O}_{3},<0.1-1.9 \mathrm{wt} \% \mathrm{Dy}_{2} \mathrm{O}_{3},<0.45 \mathrm{wt} \% \mathrm{Er}_{2} \mathrm{O}_{3}$, and $<0.16 \mathrm{Yb}_{2} \mathrm{O}_{3}$. The contents of $\mathrm{U}$ and Th vary between $2.7-5.4 \mathrm{wt} \% \mathrm{UO}_{2}$ and $8-23 \mathrm{wt} \%$ $\mathrm{ThO}_{2}$, which is equivalent to $12-39 \%$ of the cheralite component. The concentrations of $\mathrm{Si}$ were found to fluctuate around $0.5 \mathrm{wt} \% \mathrm{SiO}_{2}$, which equals $\sim 2 \%$ of the huttonite $\left(\mathrm{ThSiO}_{4}\right)$ component (Figure 4).

Members of the monazite-cheralite s.s. occur in the greisens. Here, the contents of REEs are comparatively lower (e.g., 4-17 $\mathrm{wt} \% \mathrm{Ce}_{2} \mathrm{O}_{3}$ ), while the percentage of the cheralite component simultaneously vary from 30 to $81 \%$, with the huttonite component remaining virtually unchanged. Only cheralite, instead of monazite, was deposited in the dykes of zinnwaldite granite. The contents of $45-59 \mathrm{wt} \% \mathrm{ThO}_{2}$ and $<0.1-6.4 \mathrm{wt} \% \mathrm{UO}_{2}$ represent $86-99 \%$ of the cheralite component, with the huttonite component being negligible (Figure 4 ). 

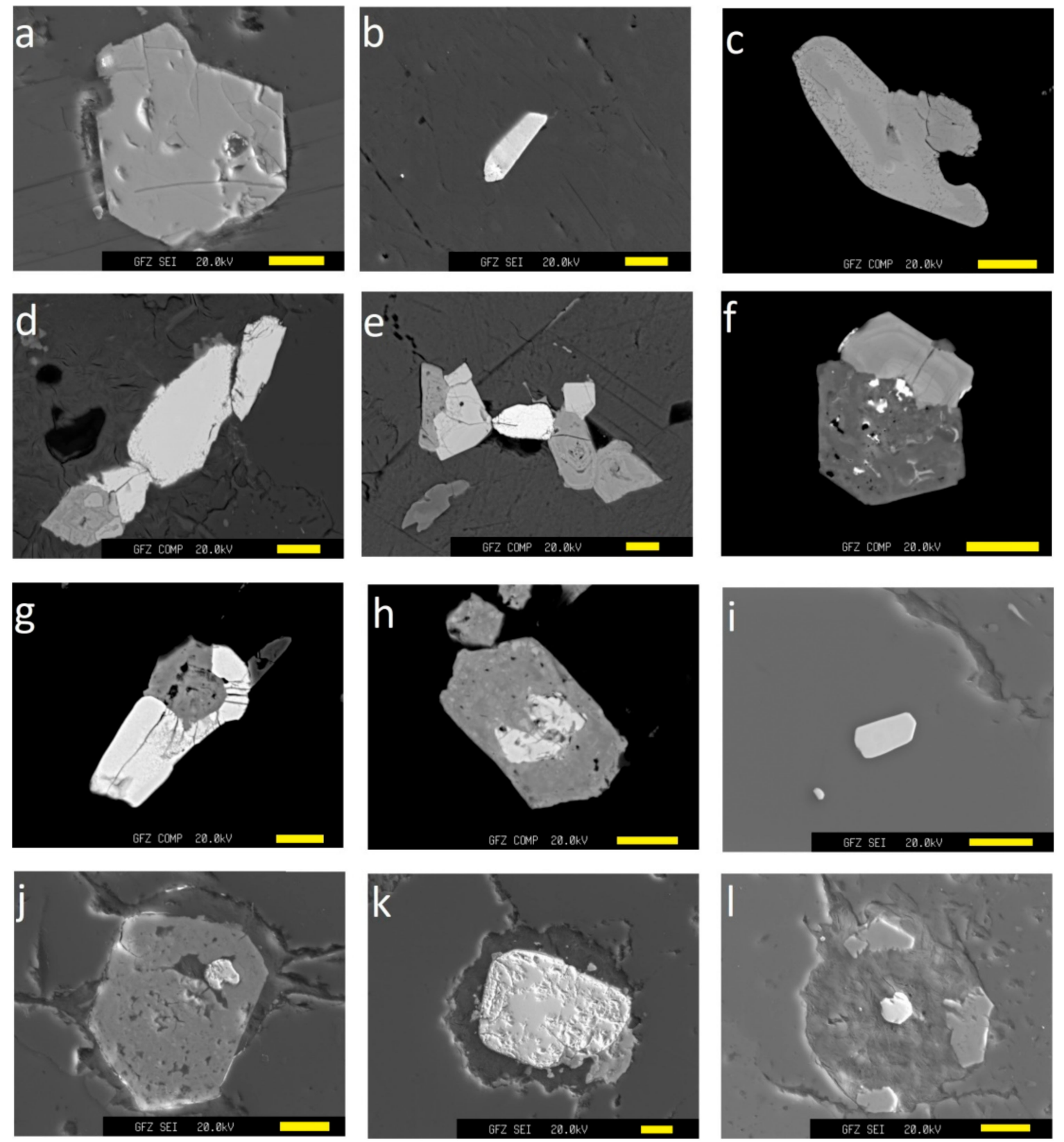

Figure 3. Back-scattered electron images of radioactive accessory minerals from Podlesí: (a) a euhedral homogeneous crystal of monazite from the deeper part of the stock granite (\#3436); (b) a columnar crystal of monazite inherited in mica in greisen (\#3365); (c) a zoned monazite crystal with rims enriched in cheralite component, deeper stock granite (\#3436); (d) three grains of monazite (white) associated with zircon (dark gray) and small grains of xenotime (light gray) from stockscheider (\#3361); (e) monazite (white in the center) associated with two grains of xenotime (light gray) and three crystals of zircon (dark gray) inherited in mica, deeper stock granite (\#3443); (f) a zoned xenotime crystal (light gray) growing on zircon (dark), deeper stock granite (\#3436); (g) xenotime (light gray) covering a zircon grain (dark), lower stock granite (\#3443); (h) xenotime (light) overgrown by zircon (dark), lower stock granite (\#3436); (i) a uraninite crystal in quartz, stockscheider (\#3361); (j) a uraninite grain in zircon, lower stock granite (\#3436); (k) a uraninite crystal rimmed by a halo of radioactively damaged feldspar, lower stock granite (\#3436); (1) a uraninite grain (white) in the center of a radioactively damaged halo in mica, three pyrite grains (dark gray) crystallized along the outer rim of the halo, lower stock granite (\#3443). Scale bar $=10 \mu \mathrm{m}$. 
Table 2. Representative results ( $\mathrm{wt} \%$ ) of electron microprobe analyses of monazite-(Ce) and monazite-cheralite s.s. from Podlesí. Formulae (in atoms per formula unit, apfu) based on 4 oxygen atoms.

\begin{tabular}{|c|c|c|c|c|c|c|c|c|c|c|}
\hline Sample & 3361 & 3361 & 3365 & 3365 & 3385 & 3385 & 3389 & 3413 & 3413 & 3436 \\
\hline $\mathrm{P}_{2} \mathrm{O}_{5}$ & 30.07 & 29.84 & 30.09 & 30.00 & 29.77 & 29.78 & 29.89 & 30.13 & 29.56 & 30.02 \\
\hline $\mathrm{SiO}_{2}$ & 0.48 & 0.40 & 0.29 & 0.34 & 0.47 & 0.42 & 0.40 & 0.21 & 0.12 & 0.39 \\
\hline $\mathrm{ThO}_{2}$ & 15.79 & 21.29 & 19.78 & 31.35 & 14.16 & 19.23 & 25.00 & 44.73 & 58.33 & 23.00 \\
\hline $\mathrm{UO}_{2}$ & 3.89 & 2.91 & 5.25 & 3.61 & 2.16 & 2.80 & 5.02 & 6.32 & 0.05 & 3.38 \\
\hline $\mathrm{Y}_{2} \mathrm{O}_{3}$ & 3.47 & 2.60 & 3.80 & 2.36 & 2.44 & 2.80 & 2.72 & 0.00 & 0.12 & 2.43 \\
\hline $\mathrm{La}_{2} \mathrm{O}_{3}$ & 6.41 & 4.78 & 4.73 & 4.71 & 5.49 & 6.04 & 2.79 & 0.94 & 0.00 & 4.53 \\
\hline $\mathrm{Ce}_{2} \mathrm{O}_{3}$ & 18.78 & 16.39 & 14.67 & 11.41 & 21.25 & 17.47 & 12.53 & 3.00 & 0.04 & 15.25 \\
\hline $\mathrm{Pr}_{2} \mathrm{O}_{3}$ & 2.31 & 2.11 & 1.88 & 1.39 & 2.86 & 2.17 & 1.82 & 0.41 & 0.00 & 2.06 \\
\hline $\mathrm{Nd}_{2} \mathrm{O}_{3}$ & 8.31 & 7.81 & 6.52 & 4.34 & 10.32 & 8.00 & 6.27 & 1.66 & 0.08 & 7.23 \\
\hline $\mathrm{Sm}_{2} \mathrm{O}_{3}$ & 2.78 & 3.18 & 2.60 & 1.55 & 3.48 & 2.62 & 3.15 & 0.41 & 0.02 & 2.67 \\
\hline $\mathrm{Gd}_{2} \mathrm{O}_{3}$ & 2.33 & 2.24 & 2.36 & 1.16 & 2.30 & 2.20 & 2.34 & 0.21 & 0.05 & 1.90 \\
\hline $\mathrm{Tb}_{2} \mathrm{O}_{3}$ & 0.38 & 0.34 & 0.41 & 0.07 & 0.31 & 0.28 & 0.35 & 0.06 & 0.00 & 0.14 \\
\hline $\mathrm{Dy}_{2} \mathrm{O}_{3}$ & 1.08 & 0.84 & 1.24 & 0.46 & 0.79 & 0.83 & 0.88 & 0.07 & 0.08 & 0.72 \\
\hline $\mathrm{Ho}_{2} \mathrm{O}_{3}$ & 0.07 & 0.05 & 0.08 & 0.04 & 0.05 & 0.09 & 0.08 & 0.00 & 0.00 & 0.08 \\
\hline $\mathrm{Er}_{2} \mathrm{O}_{3}$ & 0.26 & 0.19 & 0.24 & 0.04 & 0.16 & 0.10 & 0.11 & 0.05 & 0.00 & 0.10 \\
\hline $\mathrm{Yb}_{2} \mathrm{O}_{3}$ & 0.00 & 0.03 & 0.05 & 0.02 & 0.00 & 0.00 & 0.00 & 0.00 & 0.00 & 0.00 \\
\hline $\mathrm{CaO}$ & 3.78 & 4.66 & 5.26 & 6.79 & 3.18 & 4.15 & 6.04 & 10.63 & 11.11 & 5.17 \\
\hline $\mathrm{PbO}$ & 0.36 & 0.38 & 0.51 & 0.58 & 0.28 & 0.38 & 0.58 & 0.88 & 0.15 & 0.44 \\
\hline Total & 100.52 & 100.05 & 99.75 & 100.21 & 99.46 & 99.35 & 99.97 & 99.71 & 99.70 & 99.51 \\
\hline $\mathbf{P}$ & 0.984 & 0.985 & 0.988 & 0.986 & 0.987 & 0.987 & 0.985 & 0.994 & 0.986 & 0.991 \\
\hline $\mathrm{Si}$ & 0.018 & 0.016 & 0.011 & 0.013 & 0.018 & 0.017 & 0.016 & 0.008 & 0.005 & 0.015 \\
\hline Th & 0.139 & 0.189 & 0.175 & 0.277 & 0.126 & 0.171 & 0.222 & 0.397 & 0.523 & 0.204 \\
\hline $\mathbf{U}$ & 0.033 & 0.025 & 0.045 & 0.031 & 0.019 & 0.024 & 0.043 & 0.055 & 0.000 & 0.029 \\
\hline $\mathbf{Y}$ & 0.071 & 0.054 & 0.079 & 0.049 & 0.051 & 0.058 & 0.056 & 0.000 & 0.003 & 0.050 \\
\hline La & 0.091 & 0.069 & 0.068 & 0.067 & 0.079 & 0.087 & 0.040 & 0.014 & 0.000 & 0.065 \\
\hline $\mathrm{Ce}$ & 0.266 & 0.234 & 0.208 & 0.162 & 0.305 & 0.251 & 0.179 & 0.043 & 0.001 & 0.218 \\
\hline Pr & 0.033 & 0.030 & 0.027 & 0.020 & 0.041 & 0.031 & 0.026 & 0.006 & 0.000 & 0.029 \\
\hline Nd & 0.115 & 0.109 & 0.090 & 0.060 & 0.144 & 0.112 & 0.087 & 0.023 & 0.001 & 0.101 \\
\hline Sm & 0.037 & 0.043 & 0.035 & 0.021 & 0.047 & 0.035 & 0.042 & 0.005 & 0.000 & 0.036 \\
\hline $\mathrm{Gd}$ & 0.030 & 0.029 & 0.030 & 0.015 & 0.030 & 0.029 & 0.030 & 0.003 & 0.001 & 0.025 \\
\hline $\mathrm{Tb}$ & 0.005 & 0.004 & 0.005 & 0.001 & 0.004 & 0.004 & 0.005 & 0.001 & 0.000 & 0.002 \\
\hline Dy & 0.013 & 0.011 & 0.015 & 0.006 & 0.010 & 0.010 & 0.011 & 0.001 & 0.001 & 0.009 \\
\hline Ho & 0.001 & 0.001 & 0.001 & 0.000 & 0.001 & 0.001 & 0.001 & 0.000 & 0.000 & 0.001 \\
\hline Er & 0.003 & 0.002 & 0.003 & 0.000 & 0.002 & 0.001 & 0.001 & 0.001 & 0.000 & 0.001 \\
\hline $\mathrm{Yb}$ & 0.000 & 0.000 & 0.001 & 0.000 & 0.000 & 0.000 & 0.000 & 0.000 & 0.000 & 0.000 \\
\hline $\mathrm{Ca}$ & 0.156 & 0.194 & 0.219 & 0.282 & 0.133 & 0.174 & 0.252 & 0.444 & 0.469 & 0.216 \\
\hline $\mathrm{Pb}$ & 0.004 & 0.004 & 0.005 & 0.006 & 0.003 & 0.004 & 0.006 & 0.009 & 0.002 & 0.005 \\
\hline
\end{tabular}

b.d.l.-below detection limit of EMPA; Al, Fe, and Lu were sought but not detected.

Table 3. Representative results ( $\mathrm{w} t \%)$ of electron microprobe analyses of xenotime-( $\mathrm{Y}$ ) from Podlesí. Formulae (in atoms per formula unit, apfu) based on 4 oxygen atoms.

\begin{tabular}{cccccccccc}
\hline Sample & $\mathbf{3 3 6 1}$ & $\mathbf{3 3 6 1}$ & $\mathbf{3 3 6 1}$ & $\mathbf{3 3 8 5}$ & $\mathbf{3 3 8 5}$ & $\mathbf{3 4 3 6}$ & $\mathbf{3 4 3 6}$ & $\mathbf{3 4 4 3}$ & $\mathbf{3 4 4 3}$ \\
\hline $\mathbf{P}_{\mathbf{2}} \mathbf{O}_{\mathbf{5}}$ & 34.63 & 34.62 & 34.37 & 34.16 & 34.56 & 33.46 & 33.54 & 33.60 & 33.37 \\
$\mathbf{S i O}_{\mathbf{2}}$ & 0.50 & 0.57 & 0.58 & 0.65 & 0.48 & 1.21 & 1.19 & 0.90 & 1.17 \\
$\mathbf{T h O}_{\mathbf{2}}$ & 0.39 & 0.42 & 0.75 & 0.57 & 0.56 & 1.00 & 0.97 & 0.66 & 1.04 \\
$\mathbf{U O}_{\mathbf{2}}$ & 4.87 & 5.13 & 3.67 & 3.26 & 2.93 & 5.39 & 4.98 & 5.18 & 3.73 \\
$\mathbf{Y}_{\mathbf{2}} \mathbf{O}_{\mathbf{3}}$ & 40.66 & 39.75 & 42.35 & 42.42 & 42.80 & 40.96 & 41.49 & 41.32 & 41.28 \\
$\mathbf{C e}_{\mathbf{2}} \mathbf{O}_{\mathbf{3}}$ & 0.12 & 0.09 & 0.08 & 0.05 & 0.05 & 0.15 & 0.10 & 0.09 & 0.11 \\
$\mathbf{P r}_{\mathbf{2}} \mathbf{O}_{\mathbf{3}}$ & 0.04 & 0.05 & 0.05 & 0.03 & 0.00 & 0.04 & 0.05 & 0.04 & 0.05 \\
$\mathbf{N d}_{\mathbf{2}} \mathbf{O}_{\mathbf{3}}$ & 0.30 & 0.36 & 0.33 & 0.29 & 0.40 & 0.43 & 0.42 & 0.47 & 0.43 \\
$\mathbf{S m}_{\mathbf{2}} \mathbf{O}_{\mathbf{3}}$ & 0.42 & 0.45 & 0.45 & 0.32 & 0.43 & 0.46 & 0.41 & 0.35 \\
$\mathbf{G d}_{\mathbf{2}} \mathbf{O}_{\mathbf{3}}$ & 3.77 & 3.87 & 3.85 & 3.68 & 3.82 & 3.38 & 3.23 & 3.30 & 0.53 \\
$\mathbf{T b}_{\mathbf{2}} \mathbf{O}_{\mathbf{3}}$ & 0.98 & 0.92 & 1.04 & 0.81 & 0.81 & 0.71 & 0.84 & 0.70 & 0.75 \\
\hline
\end{tabular}


Table 3. Cont.

\begin{tabular}{|c|c|c|c|c|c|c|c|c|c|}
\hline Sample & 3361 & 3361 & 3361 & 3385 & 3385 & 3436 & 3436 & 3443 & 3443 \\
\hline $\mathrm{Dy}_{2} \mathrm{O}_{3}$ & 6.58 & 6.54 & 6.65 & 6.42 & 6.50 & 5.71 & 5.90 & 5.71 & 5.88 \\
\hline $\mathrm{Ho}_{2} \mathrm{O}_{3}$ & 0.80 & 0.79 & 0.84 & 1.04 & 0.98 & 1.01 & 0.94 & 0.88 & 0.96 \\
\hline $\mathrm{Er}_{2} \mathrm{O}_{3}$ & 2.20 & 2.03 & 2.23 & 2.72 & 2.87 & 2.71 & 2.77 & 2.62 & 2.65 \\
\hline $\mathrm{Tm}_{2} \mathrm{O}_{3}{ }^{a}$ & 0.31 & 0.27 & 0.31 & 0.33 & 0.34 & 0.36 & 0.37 & 0.35 & 0.39 \\
\hline $\mathrm{Yb}_{2} \mathrm{O}_{3}$ & 1.82 & 1.64 & 1.91 & 1.67 & 1.66 & 2.09 & 2.18 & 1.97 & 2.08 \\
\hline $\mathrm{Lu}_{2} \mathrm{O}_{3}$ & 0.33 & 0.32 & 0.32 & 0.29 & 0.29 & 0.35 & 0.30 & 0.30 & 0.37 \\
\hline $\mathrm{CaO}$ & 0.96 & 0.98 & 0.58 & 0.35 & 0.64 & 0.48 & 0.49 & 0.70 & 0.67 \\
\hline $\mathrm{PbO}$ & 0.13 & 0.17 & 0.13 & 0.17 & 0.10 & 0.25 & 0.22 & 0.00 & 0.15 \\
\hline Total & 99.81 & 98.97 & 100.49 & 99.23 & 100.22 & 100.17 & 100.40 & 99.14 & 99.11 \\
\hline $\mathbf{P}$ & 0.988 & 0.993 & 0.977 & 0.979 & 0.981 & 0.962 & 0.961 & 0.970 & 0.963 \\
\hline Si & 0.017 & 0.019 & 0.019 & 0.022 & 0.016 & 0.041 & 0.040 & 0.031 & 0.040 \\
\hline Th & 0.003 & 0.003 & 0.006 & 0.004 & 0.004 & 0.008 & 0.007 & 0.005 & 0.008 \\
\hline $\mathbf{U}$ & 0.037 & 0.039 & 0.027 & 0.025 & 0.022 & 0.041 & 0.038 & 0.039 & 0.028 \\
\hline$Y$ & 0.729 & 0.716 & 0.757 & 0.765 & 0.764 & 0.740 & 0.747 & 0.749 & 0.749 \\
\hline $\mathrm{Ce}$ & 0.001 & 0.001 & 0.001 & 0.001 & 0.001 & 0.002 & 0.001 & 0.001 & 0.001 \\
\hline Pr & 0.000 & 0.001 & 0.001 & 0.000 & 0.000 & 0.000 & 0.001 & 0.000 & 0.001 \\
\hline $\mathrm{Nd}$ & 0.004 & 0.004 & 0.004 & 0.004 & 0.005 & 0.005 & 0.005 & 0.006 & 0.005 \\
\hline Sm & 0.005 & 0.005 & 0.005 & 0.004 & 0.005 & 0.005 & 0.005 & 0.004 & 0.006 \\
\hline $\mathrm{Gd}$ & 0.042 & 0.043 & 0.043 & 0.041 & 0.042 & 0.038 & 0.036 & 0.037 & 0.040 \\
\hline $\mathrm{Tb}$ & 0.011 & 0.010 & 0.011 & 0.009 & 0.009 & 0.008 & 0.009 & 0.008 & 0.008 \\
\hline Dy & 0.071 & 0.071 & 0.072 & 0.070 & 0.070 & 0.062 & 0.064 & 0.063 & 0.065 \\
\hline Ho & 0.009 & 0.009 & 0.009 & 0.011 & 0.010 & 0.011 & 0.010 & 0.010 & 0.010 \\
\hline Er & 0.023 & 0.022 & 0.024 & 0.029 & 0.030 & 0.029 & 0.029 & 0.028 & 0.028 \\
\hline Tm & 0.003 & 0.003 & 0.003 & 0.003 & 0.004 & 0.004 & 0.004 & 0.004 & 0.004 \\
\hline $\mathrm{Yb}$ & 0.019 & 0.017 & 0.020 & 0.017 & 0.017 & 0.022 & 0.022 & 0.020 & 0.022 \\
\hline $\mathbf{L u}$ & 0.003 & 0.003 & 0.003 & 0.003 & 0.003 & 0.004 & 0.003 & 0.003 & 0.004 \\
\hline $\mathrm{Ca}$ & 0.035 & 0.036 & 0.021 & 0.013 & 0.023 & 0.017 & 0.018 & 0.026 & 0.024 \\
\hline $\mathrm{Pb}$ & 0.001 & 0.001 & 0.001 & 0.002 & 0.001 & 0.002 & 0.002 & 0.000 & 0.001 \\
\hline
\end{tabular}

${ }^{\mathrm{a}}=\mathrm{Tm}$ concentrations calculated from normalized abundances of neighboring elements $\mathrm{Dy}$ and $\mathrm{Er} ; \mathrm{Al}, \mathrm{Fe}$, and La were sought, but not detected.

Table 4. Representative results ( $w \mathrm{t} \%$ ) of electron microprobe analyses of uraninite from Podlesí. Formulae (in atoms per formula unit, apfu) based on 2 oxygen atoms.

\begin{tabular}{|c|c|c|c|c|c|c|c|c|}
\hline Sample & 3365 & 3365 & 3389 & 3436 & 3436 & 3436 & 3443 & 3443 \\
\hline $\mathrm{SiO}_{2}$ & 0.07 & b.d.l. & b.d.l. & 0.06 & 0.06 & 0.00 & 0.06 & b.d.l. \\
\hline $\mathrm{ThO}_{2}$ & 5.23 & 5.32 & 4.47 & 1.80 & 3.78 & 4.48 & 2.47 & 2.34 \\
\hline $\mathrm{UO}_{2}$ & 89.72 & 88.66 & 89.77 & 92.48 & 90.72 & 90.08 & 91.16 & 91.48 \\
\hline $\mathrm{Y}_{2} \mathrm{O}_{3}$ & 0.12 & 0.11 & 0.14 & 0.10 & 0.13 & 0.18 & 0.19 & 0.22 \\
\hline $\mathrm{Ce}_{2} \mathrm{O}_{3}$ & b.d.l. & 0.05 & b.d.l. & 0.08 & b.d.l. & 0.05 & b.d.l. & 0.02 \\
\hline $\mathrm{Nd}_{2} \mathrm{O}_{3}$ & b.d.l. & b.d.l. & 0.05 & b.d.l. & 0.02 & b.d.l. & 0.01 & b.d.l. \\
\hline $\mathrm{Sm}_{2} \mathrm{O}_{3}$ & b.d.l. & 0.15 & 0.10 & b.d.l. & b.d.l. & 0.06 & 0.03 & 0.05 \\
\hline $\mathrm{Gd}_{2} \mathrm{O}_{3}$ & b.d.l. & 0.07 & 0.05 & 0.10 & 0.02 & b.d.l. & b.d.l. & 0.09 \\
\hline $\mathrm{Tb}_{2} \mathrm{O}_{3}$ & b.d.l. & 0.01 & 0.04 & 0.05 & b.d.l. & 0.10 & b.d.l. & b.d.l. \\
\hline $\mathrm{Dy}_{2} \mathrm{O}_{3}$ & 0.05 & 0.06 & 0.09 & 0.05 & b.d.l. & 0.09 & 0.09 & 0.08 \\
\hline $\mathrm{Er}_{2} \mathrm{O}_{3}$ & 0.05 & 0.11 & 0.10 & 0.06 & 0.04 & b.d.l. & 0.03 & 0.10 \\
\hline $\mathrm{Yb}_{2} \mathrm{O}_{3}$ & 0.03 & 0.04 & b.d.l. & b.d.l. & b.d.l. & b.d.l. & b.d.l. & b.d.l. \\
\hline $\mathrm{PbO}$ & 4.13 & 4.07 & 4.05 & 4.11 & 4.07 & 3.97 & 3.97 & 3.98 \\
\hline Total & 99.41 & 98.65 & 98.86 & 98.88 & 98.83 & 99.01 & 98.01 & 98.37 \\
\hline Si & 0.003 & 0.000 & 0.000 & 0.003 & 0.003 & 0.000 & 0.003 & 0.000 \\
\hline Th & 0.054 & 0.056 & 0.047 & 0.019 & 0.040 & 0.047 & 0.026 & 0.025 \\
\hline $\mathbf{U}$ & 0.913 & 0.911 & 0.920 & 0.948 & 0.929 & 0.922 & 0.941 & 0.942 \\
\hline$Y$ & 0.003 & 0.003 & 0.003 & 0.002 & 0.003 & 0.004 & 0.005 & 0.005 \\
\hline $\mathrm{Ce}$ & 0.000 & 0.001 & 0.000 & 0.001 & 0.000 & 0.001 & 0.000 & 0.000 \\
\hline $\mathrm{Nd}$ & 0.000 & 0.000 & 0.001 & 0.000 & 0.000 & 0.000 & 0.000 & 0.000 \\
\hline
\end{tabular}


Table 4. Cont.

\begin{tabular}{ccccccccc}
\hline Sample & $\mathbf{3 3 6 5}$ & $\mathbf{3 3 6 5}$ & $\mathbf{3 3 8 9}$ & $\mathbf{3 4 3 6}$ & $\mathbf{3 4 3 6}$ & $\mathbf{3 4 3 6}$ & $\mathbf{3 4 4 3}$ & $\mathbf{3 4 4 3}$ \\
\hline $\mathbf{S m}$ & 0.000 & 0.002 & 0.002 & 0.000 & 0.000 & 0.001 & 0.000 & 0.001 \\
$\mathbf{G d}$ & 0.000 & 0.001 & 0.001 & 0.001 & 0.000 & 0.000 & 0.000 & 0.001 \\
$\mathbf{T b}$ & 0.000 & 0.000 & 0.001 & 0.001 & 0.000 & 0.002 & 0.000 & 0.000 \\
$\mathbf{D y}$ & 0.001 & 0.001 & 0.001 & 0.001 & 0.000 & 0.001 & 0.001 & 0.001 \\
$\mathbf{E r}$ & 0.001 & 0.002 & 0.001 & 0.001 & 0.001 & 0.000 & 0.000 & 0.001 \\
$\mathbf{Y b}$ & 0.000 & 0.001 & 0.000 & 0.000 & 0.000 & 0.000 & 0.000 & 0.000 \\
$\mathbf{P b}$ & 0.051 & 0.051 & 0.050 & 0.051 & 0.050 & 0.049 & 0.050 & 0.050 \\
\hline
\end{tabular}

b.d.l. = below detection limit, $\mathrm{P}$ was sought, but not detected.

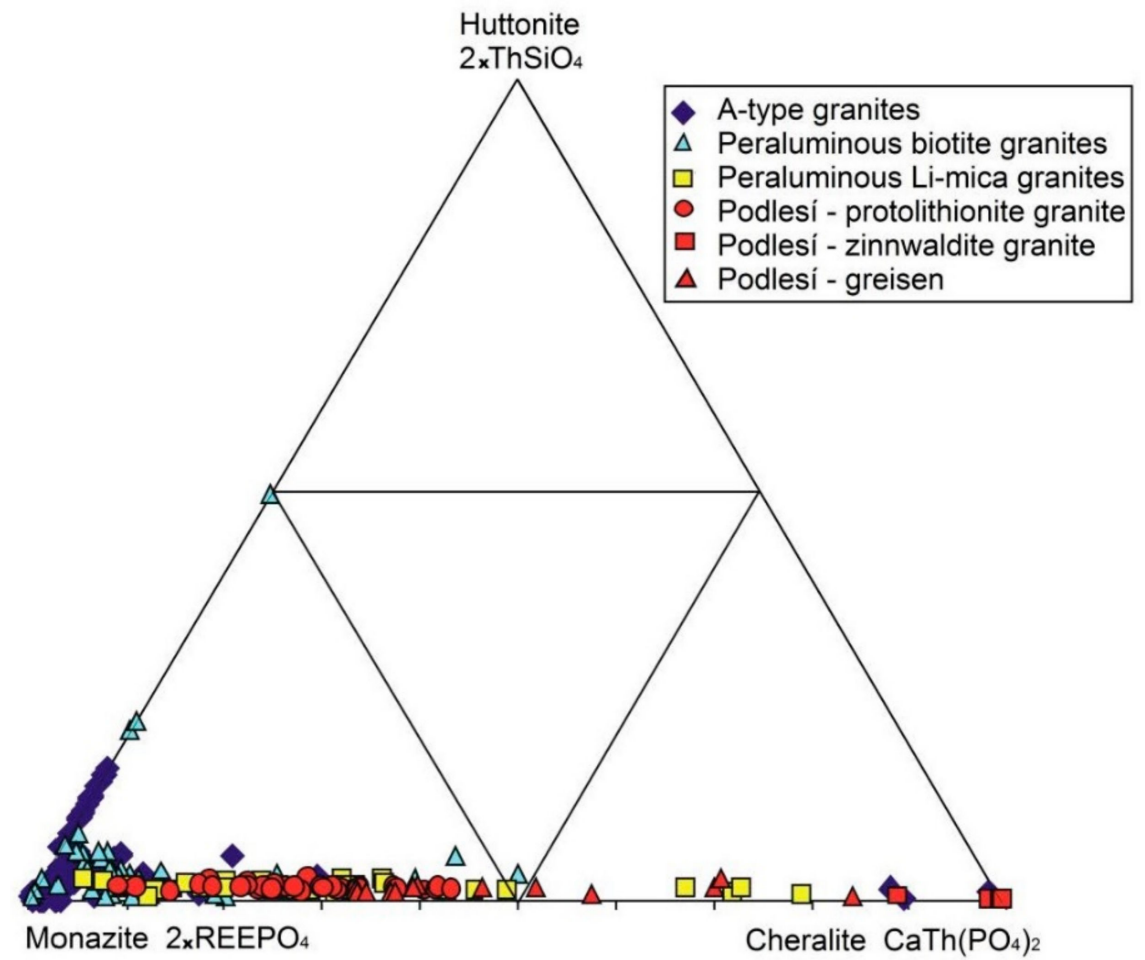

Figure 4. The monazite-cheralite-huttonite classification diagram after [25].

Li-biotite granite at the exocontact of the Podlesí stock contains two chemical populations of monazite. One is identical with that described from protolithionite granite, while the other is poor in Th and $\mathrm{U}$ (5.5-9.5 $\mathrm{wt}^{\mathrm{T}} \mathrm{ThO} \mathrm{ThO}_{2}$, equaling to $4-9 \%$ cheralite) but rich in $\mathrm{La}$ and $\mathrm{Ce}$ (11-15 wt \% $\left.\mathrm{La}_{2} \mathrm{O}_{3}, 30-33 \mathrm{wt} \% \mathrm{Ce}_{2} \mathrm{O}_{3}\right)$, i.e., close to monazite-(Ce) endmember composition.

Chondrite-normalized REE patterns display, typically for monazite, a strong LREE enrichment with a deep negative $\mathrm{Eu}$ anomaly and predominantly moderate negative anomalies at $\mathrm{La}$ and $\mathrm{Nd}$ in all varieties of the protolithionite granite (Figure $5 \mathrm{a}, \mathrm{b}$ ). Monazitecheralite s.s. from zinnwaldite granite and greisens are LREE-poor, with generally flat patterns and distinct kinks at $\mathrm{La}$ and $\mathrm{Nd}$. Monazite from the surrounding Li-biotite granite is LREE-rich, with the majority of grains showing relatively low contents of La (Figure 5c). 

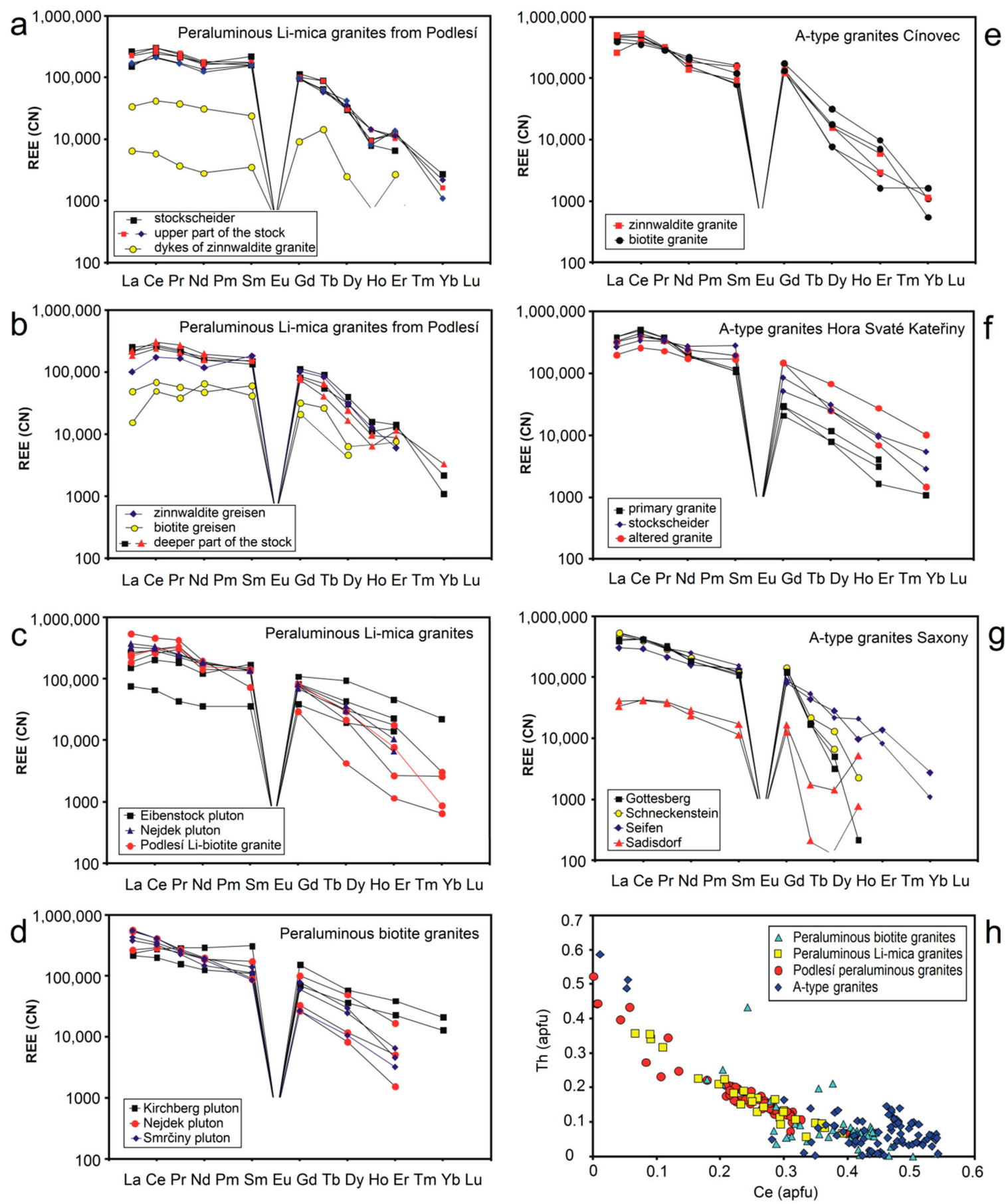

Figure 5. Chondrite-normalized [26] rare earth element (REE) distribution patterns and Th contents in monazite from the Erzgebirge: (a-d) REE patterns of monazite from the peraluminous suite; (e-g) REE patterns of monazite from the A-suite; (h) Ce vs. Th in monazite from all types of granites.

\subsection{Xenotime- $(Y)$}

Xenotime is rare in the protolithionite granite and very rare in the zinnwaldite granite dykes, which is in accordance with the extremely low contents of $Y$ and HREE in these granites. Xenotime grains are usually spatially associated with zircon, and zircon-xenotime intergrowths are common, reflecting their corresponding crystal structure (Figure $3 \mathrm{e}-\mathrm{h}$ ). Xenotime crystals show only rarely an oscillatory zoning in BSE images (Figure 3f), and the majority of grains are chemically homogeneous. In association with zircon, xenotime almost always precipitated later (Figure $3 \mathrm{f}, \mathrm{g}$ ) and is only rarely older (Figure $3 \mathrm{~h}$ ). Xenotime 
from all rock varieties is $\mathrm{Y}$ - and MREE-dominant, containing 39.4-43.0 wt $\% \mathrm{Y}_{2} \mathrm{O}_{3}, 2.2-$ $4.1 \mathrm{wt} \% \mathrm{Gd}_{2} \mathrm{O}_{3}, 5.4-6.9 \mathrm{wt} \% \mathrm{Dy}_{2} \mathrm{O}_{3}$, and 2.0-2.9 $\mathrm{wt} \% \mathrm{Er}_{2} \mathrm{O}_{3}$ (Table 3, Figure 6a). The concentrations of $\mathrm{U}$ reach $2.7-5.4 \mathrm{wt} \% \mathrm{UO}_{2}$, while Th is low $\left(<1 \mathrm{wt} \% \mathrm{ThO}_{2}\right)$.
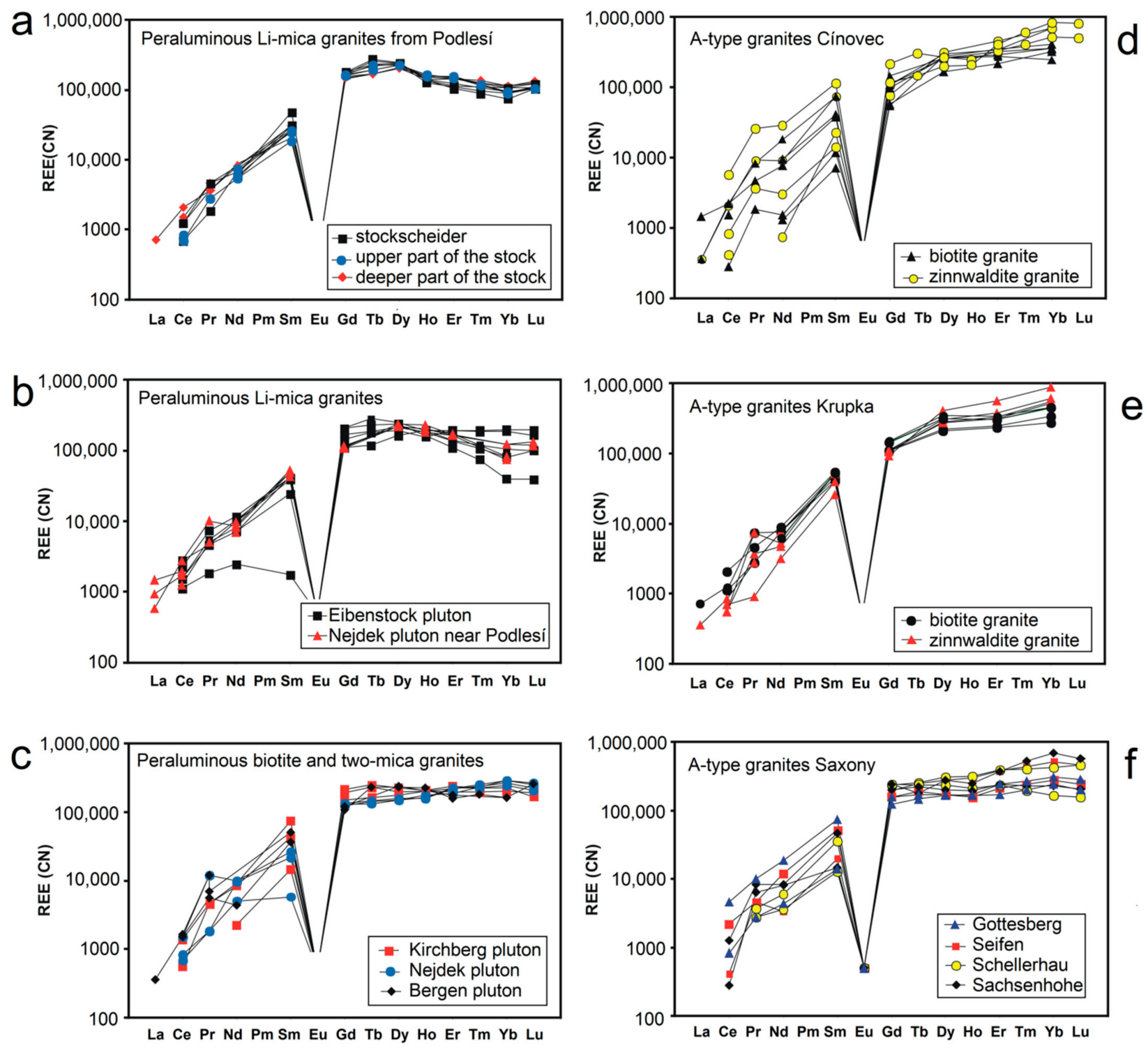

Figure 6. Chondrite-normalized [26] REE distribution patterns of xenotime from the Erzgebirge. (a-c) granites of the peraluminous suite; $(\mathbf{d}-\mathbf{f})$ granites of the A-suite.

Xenotime from the surrounding Li-biotite granite is somewhat more Y-rich (40.0$46.7 \mathrm{wt} \% \mathrm{Y}_{2} \mathrm{O}_{3}$ ); its REE pattern is also MREE-dominant (Figure 6b). The concentrations of $\mathrm{U}$ and $\mathrm{Th}$ resemble those in xenotime in the other granite varies, i.e., $2.8-4.8 \mathrm{wt} \% \mathrm{UO}_{2}$ and $0.5-1.1 \mathrm{wt} \% \mathrm{ThO}_{2}$.

\subsection{Uraninite}

Uraninite constitutes a common accessory mineral in the deeper part of the protolithionite granite and rarely occurs also in greisens. It forms small, homogeneous, subhedral-to-euhedral crystals enclosed in quartz (Figure 3i), zircon (Figure 3j), and most often mica (Figure 3k,l). In mica, uraninite is typically surrounded by a "pleochroic halo", reflecting the damaged crystal structure of the enclosing mica due to irradiation by $\alpha$ particles. In this halo, pyrite is often precipitated as a secondary mineral (Figure 31). 
The chemical composition of uraninite is simple: in addition to uranium (88.7-91.5 $\mathrm{wt} \%$ $\left.\mathrm{UO}_{2}\right)$ and thorium $\left(1.5-5.3 \mathrm{wt} \% \mathrm{ThO}_{2}\right)$, it only contains some $\mathrm{Y}\left(<0.22 \mathrm{wt} \% \mathrm{Y}_{2} \mathrm{O}_{3}\right)$, heavy REEs $\left(<0.5 \mathrm{wt} \% \mathrm{HREE}_{2} \mathrm{O}_{3}\right)$, and $\mathrm{Pb}(4.0-4.2 \mathrm{wt} \% \mathrm{PbO}$, originating as a result of the radioactive decay of $U$ and $T h$ ) (Table 4 ). There is no difference in chemistry between uraninites from different rock types within the Podlesí stock (Figure 7).
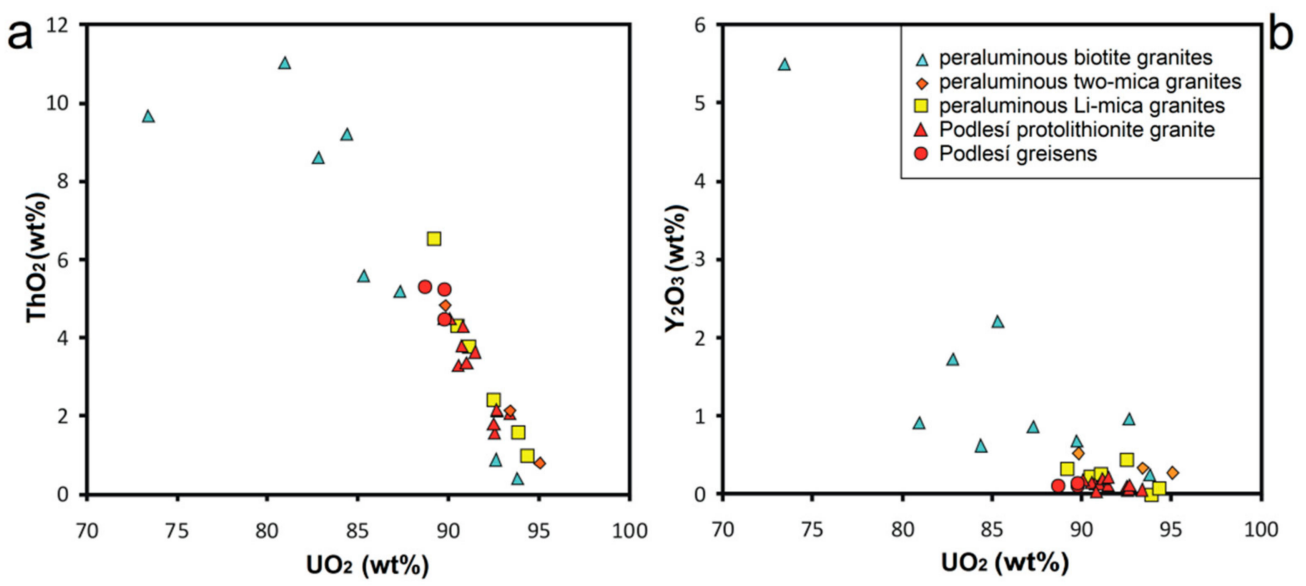

Figure 7. Chemical composition of uraninite. (a) $\mathrm{UO}_{2}$ vs. $\mathrm{ThO}_{2} ;\left(\right.$ b) $\mathrm{UO}_{2}$ vs. $\mathrm{Y}_{2} \mathrm{O}_{3}$.

\section{Discussion}

5.1. Evolution of Monazite and Xenotime Compositions during Magmatic/Hydrothermal Evolution of the Podlesi Granite System

The Podlesí granite stock represents one of the most extremely fractionated; peraluminous; F-, Li-, and P-rich; and Sn-, $\mathrm{W}-$, Nb-, and Ta-bearing rare-metal granite system worldwide. In contrast to other geochemically comparable granite plutons, like Argemela, Portugal [27]; Beauvoir, France [28]; and Ehrenfriedersdorf [29] or Yichun, China [30]; the Podlesí stock evolved as a repeatedly opened system characterized by non-equilibrium conditions. Explosive breccia, stockscheider, magmatic layering, unidirectional solidification textures (UST), and late dykes with intra-dyke brecciation are the most important features of this granite occurrence. Therefore, Podlesí should be discussed as a transitional member, connecting the classical rare-metal granite plutons with subvolcanic Sn-Mo porphyry systems [31].

Disturbed and non-equilibrium conditions during the crystallization of magma also influenced the evolution of U-Th-bearing accessory minerals. Nearly constant U/Th values ( 1:4 apfu, Figure 8a) in the majority of monazite grains within the Podlesí stock indicate that these two elements were not separated in the course of late magmatic to post-magmatic processes. On the other hand, the Y/Ce value and the absolute concentrations of Th and U varied substantially. Monazite from chemically less evolved Li-biotite granite is Th-poor and relatively La-rich, while monazite from the more evolved protolithionite granites and greisens is rich in $T h+U$ (cheralite component) and poor in $(Y+R E E)$ (Figure 9). Cheralite of near-endmember composition crystallized at the base of the major dyke of zinnwaldite granite, where the deposition of $\mathrm{Nb}$-Ta oxides indicates a strong influence of metalliferous hydrothermal fluids [32]. 

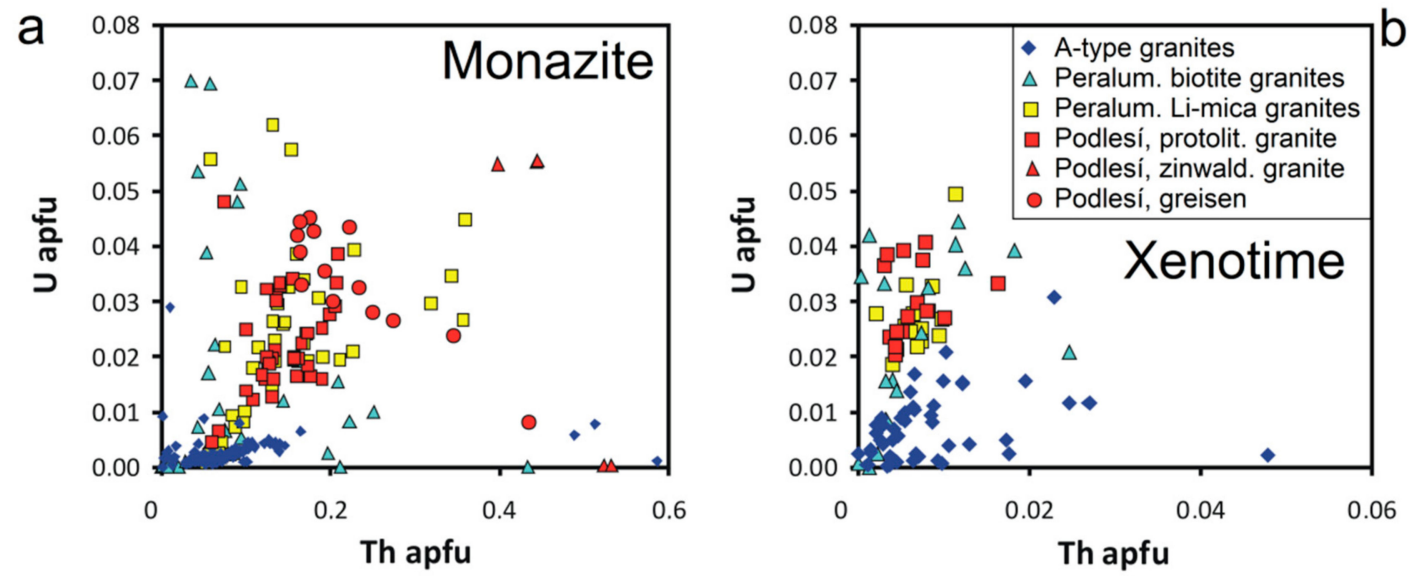

Figure 8. U/Th values in minerals. (a) monazite; (b) xenotime.

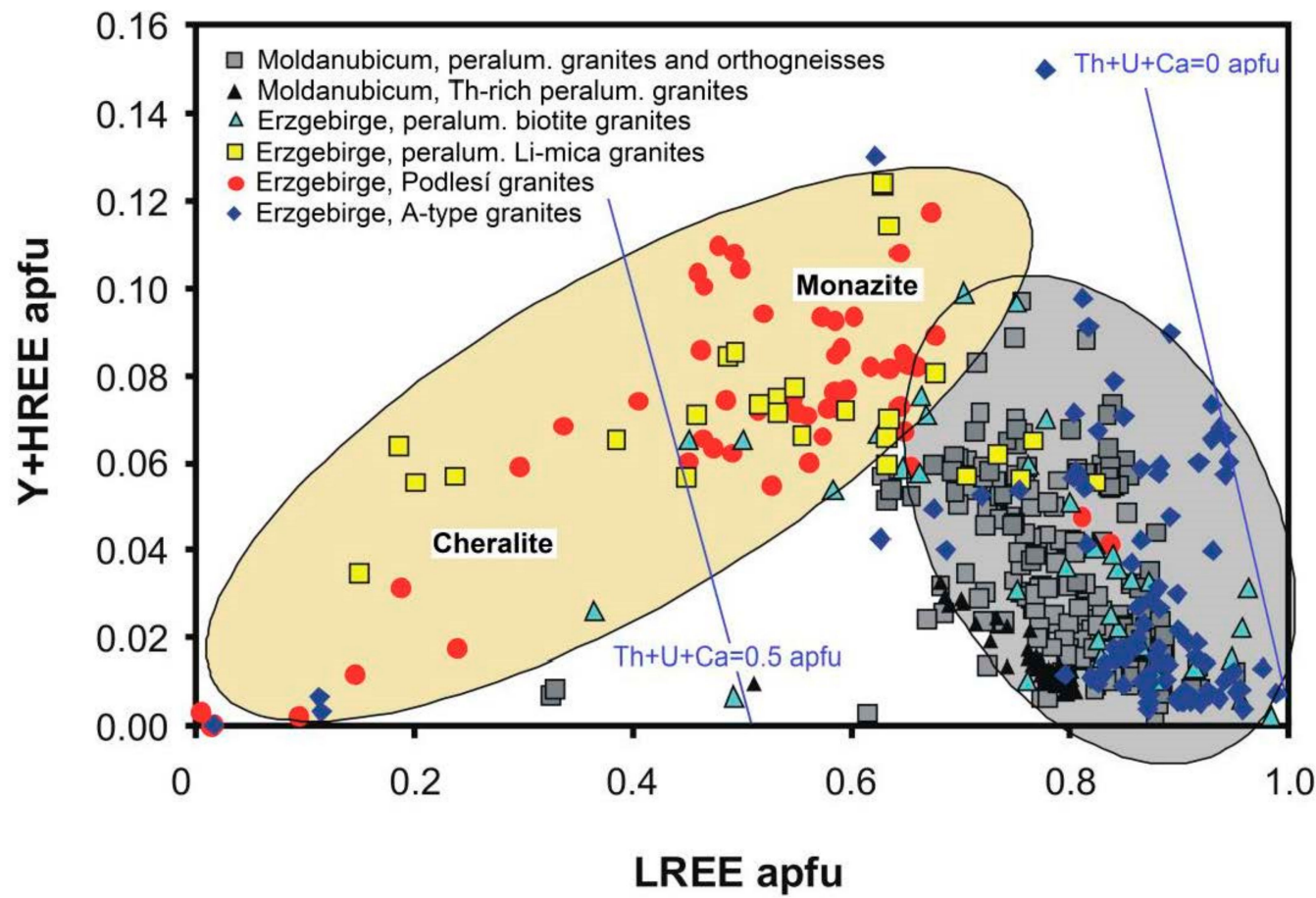

Figure 9. Light rare earth elements (LREE) vs. Y + HREE (heavy rare earth elements) contents in monazite of different origins. Data from the Moldanubicum are taken from [5]. Blue lines visualize identical contents of $\mathrm{Th}+\mathrm{U}+\mathrm{Ca}$ (huttonite + cheralite component). Gray field: Th,U-poor monazite from A-type and less fractionated S-type granites. Beige field: Th, U-rich monazite-cheralite s.s. from fractionated S-type granites.

Xenotime displays minor differences in chemistry among the individual granite facies. Nevertheless, a small difference between the Podlesí stock and the surrounding older Li-biotite granite is recognizable, with the former being relatively rich in Gd (3.3-4.1 vs. $\left.2.0-2.5 \mathrm{wt} \% \mathrm{Gd}_{2} \mathrm{O}_{3}\right)$, and the latter in $\mathrm{Y}\left(44-47 \mathrm{vs} .39-43 \mathrm{wt} \% \mathrm{Y}_{2} \mathrm{O}_{3}\right)$ and $\mathrm{Ho}(1.1-1.4 \mathrm{vs}$. $0.8-1.0 \mathrm{wt} \% \mathrm{Ho}_{2} \mathrm{O}_{3}$ ). The contents of Th and $\mathrm{U}$, with maxima at $5.4 \mathrm{wt} \% \mathrm{UO}_{2}$ and $1.1 \mathrm{wt} \%$ $\mathrm{ThO}_{2}$ are scattered, showing no correlation. Intermediate solid solutions in the xenotimezircon-thorite-coffinite system, frequently observed in mineralized members of the granite group \#4 in the eastern Erzgebirge [8,14] did not form at Podlesí. Notably, xenotime from all facies shows high $\mathrm{Y} / \mathrm{Ho}$ ratios between 37 and 46 , which broadly resemble the ratios of 33-41 determined for their host rocks [23]. 


\subsection{Diversity of Monazite and Xenotime Compositions among Granite Groups}

Since uraninite data are scarce and only available for granite groups \#1-3, we have restricted the comparison to monazite and xenotime. Available data permit comparing the composition of these two species (i) between less and more fractionated rocks, and (ii) between rocks of S- and A-type affiliation.

Monazite, due to its crystal structure, prefers light trivalent REEs [33]. Lanthanum, Ce, $\mathrm{Pr}$, and $\mathrm{Nd}$ are usually the dominant constituents, but their ratios may vary broadly. Based on the shape of the chondrite-normalized REE distribution pattern (Figure $5 \mathrm{a}-\mathrm{g}$ ) and the contents of Th (Figure 5i), three types of monazite can be distinguished in the Erzgebirge (Figure 10):

1. Th-poor monazite with a steep but smooth REE distribution pattern gradually declining from La to $\mathrm{Gd}$ and devoid of anomalies, with a distinct negative Eu anomaly and very low contents of the HREE. This pattern characterizes less fractionated biotite granites of both S and A types (Nejdek-Eibenstock pluton, Schneckenstein, Gottesberg, lower part of the Cínovec cupola) and, occasionally, also less evolved intrusions of the peraluminous Li-mica granite group. This may be interpreted as a product of magmatic crystallization from relatively F-poor melt without or with only low-grade fluid-melt and/or fluid-crystals reaction.

2. Th-poor monazite with REE patterns similar to previous type 1 but with maxima at $\mathrm{Ce}$ and Pr occurs in more evolved A-type granite facies at Cínovec and Hora Svaté Kateřiny.

3. Th-rich monazite with relatively lower and flat LREE pattern from La to Sm, again with maxima at $\mathrm{Ce}$ and $\mathrm{Pr}$, typifies peraluminous Li-mica granites including the most fractionated member of this group, the Podlesí stock. In these rocks, the relatively lower sum of REEs is compensated by enrichment in Th.

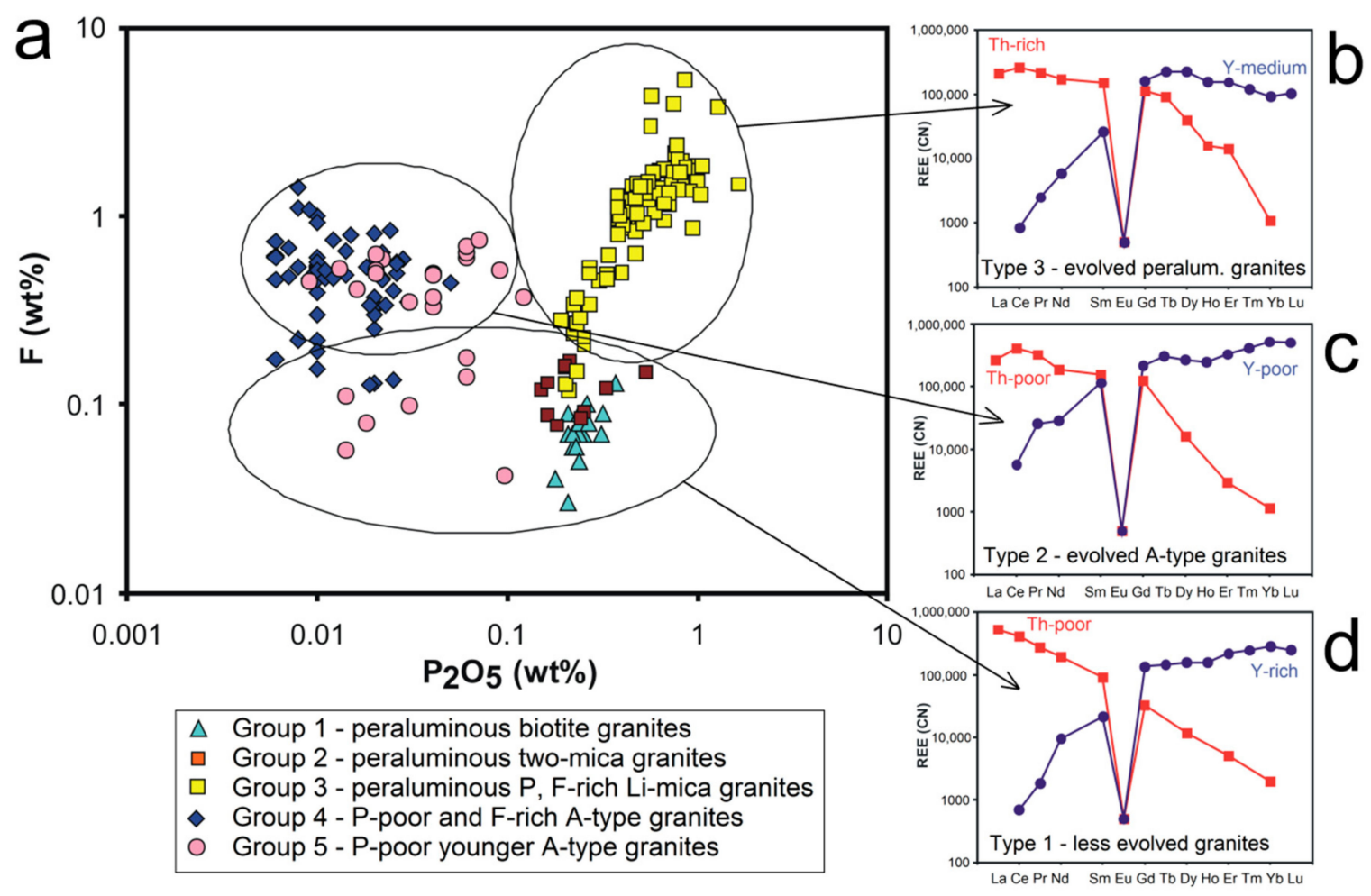

Figure 10. Chemical composition of Erzgebirge granites in terms of F versus $\mathrm{P}_{2} \mathrm{O}_{5}(\mathbf{a})$ and the related typical chondritenormalized REE patterns of monazite and xenotime $(\mathbf{b}-\mathbf{d})$. Red patterns-monazite; blue patterns-xenotime. Classification of granites according to [12], bulk-rock data from $[9,12,14,19,23,34]$ and unpublished data. See text for details. 
Generally, the shape of the monazite REE patterns with the observed anomalies corresponds to the shape of the REE patterns in the parental rocks [6,34,35]. In all cases, the parental rocks of these monazites underwent an intensive late/post-magmatic reaction with F-rich fluids.

Occasionally, peraluminous Li-mica granites contain monazite grains with both Type 1 and Type 3 of REE patterns. This can be probably explained by the occurrence of two populations (early-late) or may reflect the presence of primary magmatic and late- to post-magmatically altered grains.

Substitution of Th in monazite is different in S- and A-type granites. Monazite from peraluminous granites of groups \#1-3, typically in the Podlesí stock and the NejdekEibenstock pluton, forms a continuous solid-solution series with cheralite [25], with only a very small contribution of the huttonite component (Figure 4). At the content of ca. $24 \mathrm{wt} \%$ $\mathrm{ThO}_{2}$, the cheralite component approaches $50 \%$. The only exception with a dominant monazite-huttonite solid solution is F-poor biotite granites (group \#1), forming the Kirchberg massif [6]. In contrast, the cheralite substitution is limited (max. $0.2 \mathrm{apfu}$ ), and the huttonite component dominates in monazites from the A-type granites.

The contents of $U$ are substantially higher in monazite from peraluminous granites (up to $0.07 \mathrm{apfu} \mathrm{U})$ than in A-type monazites ( $<0.01 \mathrm{apfu} \mathrm{U}$, Figure $8 \mathrm{a})$. There is no systematic $\mathrm{U} / \mathrm{Th}$ correlation.

Yttrium in monazite is usually less than 0.06 apfu (only exceptionally up to $0.11 \mathrm{apfu}$ ), which is consistent with equilibrium crystallization temperatures of about $600{ }^{\circ} \mathrm{C}[36,37]$. The ( $\mathrm{Y}+$ HREE) contents reach max. 0.15 apfu (Figure 11), with a Y/HREE ratio of about 4:1 in peraluminous granites and mostly 2-3:1 in A-type granites. Monazite from less evolved peraluminous granites and all A-type granites is generally Th-poor, and the sum of LREE + HREE + Y approaches 1 apfu. Thus, the correlation between LREE and HREE $+\mathrm{Y}$ is negative (gray field in Figure 9). Monazite from more evolved peraluminous rocks is Th-rich: along with the increase in the cheralite component, LREE and HREE $+\mathrm{Y}$ are substituted proportionally; i.e., the correlation LREE vs. HREE + Y becomes positive (light blue field in Figure 9).

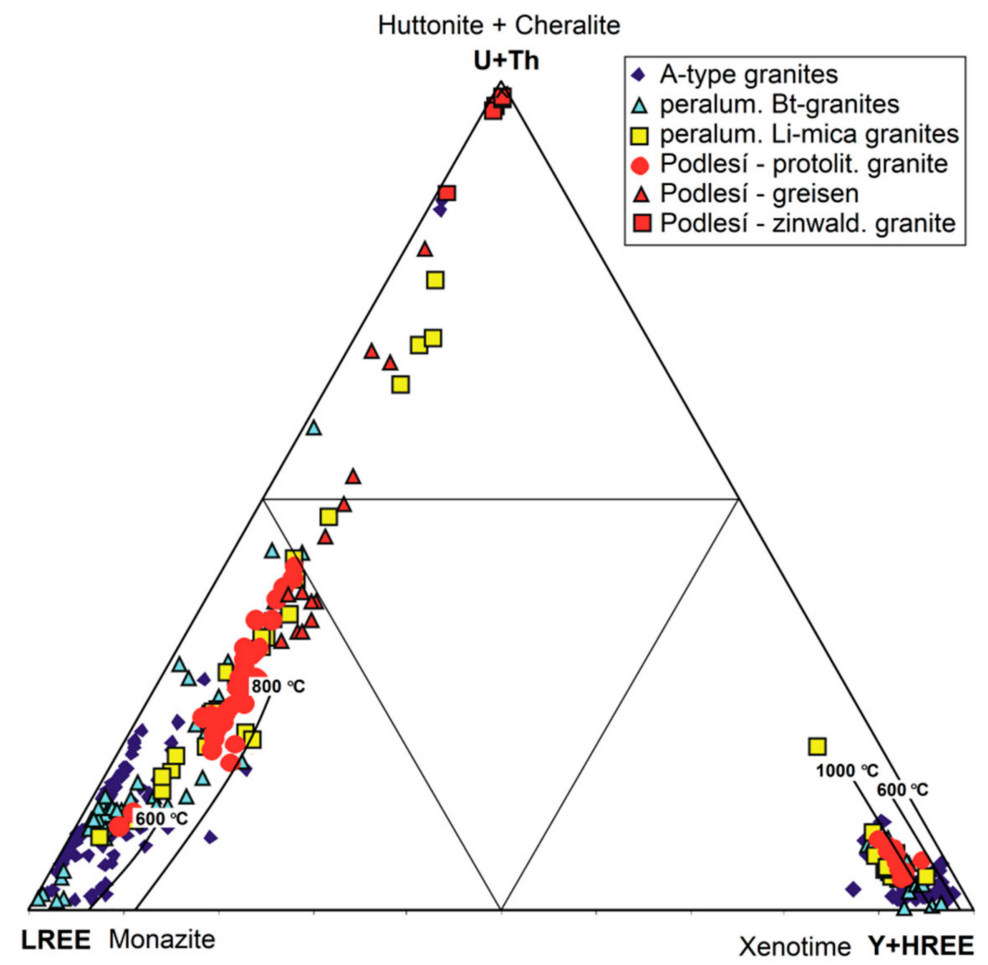

Figure 11. The monazite-xenotime-huttonite classification diagram. Temperatures of co-existing monazite-xenotime pairs in ${ }^{\circ} \mathrm{C}$ according to [36,37]. 
The tetragonal crystal structure of xenotime prefers, besides $Y$, the heavy REE. Much like monazite, xenotime can be discriminated into three types according to its chondritenormalized REE patterns:

1. Xenotime with a flat MREE-HREE pattern characterizes less fractionated biotite granites of both S- and A-type affiliation. This xenotime is mostly Y-rich, containing 0.80-0.85 apfu Y. Y/Ho ratios are generally near-chondritic (26-34) in less evolved rocks, but also may be superchondritic (up to 55) in more fractionated group 12 granites from Kirchberg and Bergen.

2. Xenotime, with a smoothly inclining, $\mathrm{Yb}$-Lu-dominated chondrite-normalized REE pattern defines fractionated A-type granites. At the same time, this type of xenotime is the most Y-poor one among all analyzed xenotime varieties. Xenotime exhibiting the lowest $Y$ content (0.4-0.6 apfu Y) was discovered immediately below the upper contact of mineralized cupolas of A-type granites in the eastern Erzgebirge. Fractionated granites in the eastern Erzgebirge frequently contain xenotime possessing subchondritic $\mathrm{Y} / \mathrm{Ho}$ ratios (down to 14).

3. MREE-dominant (Gd-Tb-Dy) xenotime with $0.75-0.83$ apfu $\mathrm{Y}$ is typical of peraluminous Li-mica granites of group \#3. Most evolved representatives of this group are distinguished by suprachondritic $\mathrm{Y} / \mathrm{Ho}$ ratios (up to 44).

The contents of Th in xenotime are minor in all studied granites and distinctly lower than in monazite, while the contents of $U$ are similar in both species. The concentrations of $\mathrm{U}$ tend to be slightly higher in xenotime from peraluminous granites (0.02-0.05 apfu U) relative to that crystallized in A-type granites ( $<0.02 \mathrm{apfu} U)$, while Th in xenotime from both granite types is almost identical (mostly $<0.025$ apfu Th) (Figure $8 b$ ).

One explanation of the described particular chemical signatures of monazite and xenotime in the various types of granites lies in the composition of the parental rocks and in the association and timing of crystallization of accompanying accessories, especially zircon (host of Th, HREE, and Y), fluorite (sink for Y and REE), apatite (REE), and thorite (Th, U, REE). Subaluminous A-type rocks are relatively Ca-poorer and Si-richer compared to peraluminous granites, which may support the crystallization of thorite and substitution of $(\mathrm{Th}+\mathrm{U})$ as the huttonite component in monazite. Under strongly peraluminous conditions, thorite and huttonite seem to be unstable, and Th becomes incorporated as the cheralite component in monazite or, finally, may account for the precipitation of cheralite sensu stricto. The distribution of REEs between monazite and xenotime (Figure 11) may serve as a geothermometer: in peraluminous granitic melts, contents of about $5 \%$ of the monazite component in xenotime indicate crystallization at $600-900{ }^{\circ} \mathrm{C}$, while $6-15 \%$ of the xenotime component in monazite imply temperatures of roughly $600-800{ }^{\circ} \mathrm{C}$ [36]. These data would be in line with the crystallization of the (monazite + xenotime) pairs discussed in this paper from the granitic melts or at the magmatic/hydrothermal transition. Generally, very low contents of the xenotime component in monazite from P-poor A-type rocks and some peraluminous biotite granites imply crystallization temperatures as low as ca. $450{ }^{\circ} \mathrm{C}$ [37], but this temperature estimate is likely too low and disturbed by the earlier crystallization of $(Y+$ HREE)-substituting zircon, xenotime, and thorite (in A-type rocks also fluorite), i.e., reflects monazite crystallization from an already Y-depleted melt. Temperatures of $>1000{ }^{\circ} \mathrm{C}$, indicated by $5-7 \%$ monazite component in some xenotime grains (Figure 11), are unrealistically high and probably reflect the difference between equilibria reached under experimental conditions [36] and in real volatile-rich melt.

As noted previously, many of the monazite grains from various facies of the Podlesí system as well as other evolved, mineralized representatives of group \#3-4 granites display chondrite-normalized LREE patterns with minima at La and Nd [6]. Xenotime from these rocks shows corresponding kinks at $\mathrm{Ho}$ and, occasionally, also $\mathrm{Lu}$, and simultaneously non-chondritic and either super- or suprachondritic $Y$ /Ho values [7]. This special type of REE pattern is usually termed the lanthanide tetrad effect. This effect is well-known from fractionated S- and A-type granites elsewhere and from genetically related pegmatites, 
including those in the study area [2,3] but has also been reported from other accessory phases, such as zircon [38] and apatite [39].

The origin of this pattern or fractionated $\mathrm{Y} / \mathrm{Ho}$ ratios in rocks and minerals is a matter of debate. Many authors suppose a model involving the presence of volatile (F)-rich melts or fluids as an indispensable prerequisite for the formation of these features, e.g., [40-43]. Using the tetrad effect terminology and quantification procedure proposed by Irber [41], the first tetrad T1 from monazite falls mostly within the interval of 1.0-1.4, while the third tetrad from xenotime is typically in the range 1.0-1.6.

The aforementioned fluid-related model has been recently questioned by [44], who demonstrated experimentally that the tetrad effect could well originate in F-free peraluminous granitic melts in the absence of any fluids, predominantly triggered by monazite and xenotime fractionation. Regarding the Podlesí and similar rocks in the study area, they are both strongly fractionated and volatile (F)-rich. Thus, it cannot be unambiguously clarified whether coupled (monazite + xenotime) fractionation or melt/fluid interactions are responsible for the origin of these features or both operated in concert.

It is worth noting that none of the three types of monazite from the Erzgebirge show a positive Ce anomaly, common in rocks hydrothermally altered by oxidizing solutions [45]. The small relative enrichment of Ce in comparison with La in the REE-patterns of type 2 and 3 monazites (Figure 10) are more likely related to the tetrad effect, since geochemically more evolved rocks of groups \#3-5 crystallized at low oxygen fugacity, close to or below the QFM buffer [46].

\subsection{Comparison with Other Areas}

A comprehensive study of accessory minerals from peraluminous two-mica granites and peraluminous biotite- and two-mica orthogneisses from the Moldanubicum [5] (Figure 9) has shown that monazite from "common" peraluminous rock is, with rare exceptions, rich in LREE ( $>0.9$ LREE apfu) and poor in Y, HREE, and (Th + U). The only exceptions within the Moldanubicum are two small bodies of Th-rich (up to $100 \mathrm{ppm}$ Th in bulk rock) granites at Gutau [47] and Dreiländereck [48], containing monazite with ca. 0.2 apfu $(\mathrm{Th}+\mathrm{U})$. Cheralite substitution strongly prevails over the huttonite one in monazite from all these peraluminous rocks. The strong dominance of the cheralite substitution mechanism in peraluminous granites has also been stressed from Western Carpathians [49-51], from the Slavkovský Les area [52], from the Fichtelgebirge [53], and also for Alpine orthogneisses [54] and the strongly peraluminous and perphosphorous Belvís de Monroy pluton in the Iberian Variscan belt [55]. In contrast, Reference [56] established the huttonite substitution as dominant in both magnetite- and ilmenite-series granites from a subduction-related magmatic arc in Japan. The same observation was made by [4] in monazite from the A-type Erongo granite from Namibia.

Regarding the evolution of the REE pattern in the course of magma fractionation, Reference [56] corroborated our observation about a relative flattening of the REE curves with an increasing fractionation in composite plutons of all geochemical types.

\section{Concluding Remarks}

In granitic rocks, monazite and xenotime are crucial for the whole-rock budgets of the REE and Th. Their chondrite-normalized REE patterns largely mimic that of their parental rock with respect to the shape and the presence of anomalies. Compositional peculiarities of these two accessory species are a function of the chemistry of the granite host, its degree of magmatic fractionation, and the intensity of late magmatic to post-magmatic hydrothermal alteration that the host may have experienced.

Monazite-cheralite solid solutions (s.s.) and xenotime from the highly evolved, strongly peraluminous, P-F-Li-rich Podlesí granite stock indicate that, with the increasing degree of magmatic and high-T early post-magmatic evolution, the content of the cheralite component in monazite increases and the relative dominance of the MREE in xenotime becomes larger. Considering the overall compositional signatures of these two accessory 
minerals in the late Variscan granites of the Erzgebirge/Krušné Hory Mts., three types of granites can be distinguished: (i) chemically less evolved F-poor granites of both S(I)- and A-type affiliation contain monazite with a smooth, mostly symmetric chondrite-normalized (CN) REE pattern gradually declining from La to Gd; associated xenotime is Y-rich $(>0.8$ apfu Y) with a flat MREE-HREE pattern; (ii) fractionated A-type granites typically contain La-depleted monazite with Th accommodated as the huttonite component, combined with usually Y-poor (0.4-0.6 apfu Y) xenotime characterized by a smoothly inclining, HREE ( $\mathrm{Yb}-\mathrm{Lu}$ )-dominant $\mathrm{CN}$-REE pattern; (iii) fractionated peraluminous Li-mica granites host monazite with a flat, asymmetric (kinked at La and Nd) LREE pattern; associated xenotime is distinctly MREE (Gd-Tb-Dy)-dominant. Monazite and xenotime account for the bulk of the REE budgets in all types of granite. In peraluminous S(I)-type granites free of thorite, almost all Th is accommodated in monazite-cheralite s.s In contrast, Th budgets in A-type granites are accounted for by monazite-huttonite s.s. together with thorite. Uraninite, if present, is the most important carrier of $U$ in peraluminous rocks. In the A-type granites, uraninite is rare or does not precipitate. In these rocks, the $\mathrm{U}$ budget was predominantly controlled by monazite and xenotime.

Supplementary Materials: The following are available online at https://www.mdpi.com/2075-1 63X/11/2/127/s1: Supplementary Electronic Material S1: Bulk-rock chemical composition of the studied samples. Supplementary Electronic Material S2: Electron microprobe analyses of the studied minerals from Podlesí.

Author Contributions: The whole project, starting with laboratory work and ending with final editing of the manuscript, represents a joint work of both authors. Both authors have read and agreed to the published version of the manuscript.

Funding: This study was supported by the Czech Science Foundation, project No. P210/19/05198S, and by RVO 67985831 of the Institute of Geology of the Czech Academy of Sciences.

Institutional Review Board Statement: Not applicable.

Informed Consent Statement: Not applicable.

Data Availability Statement: The data presented in this study are available in electronical Supplementary Materials S1 and S2.

Acknowledgments: Dieter Rhede and Oona Appelt (GFZ Potsdam) and Radek Škoda (Masaryk University Brno) are thanked for their support with the electron microprobe work. Reviewers are thanked for their valuable and constructive reviews.

Conflicts of Interest: The authors declare no conflict of interest.

\section{References}

1. Bea, F. Residence of REE, Y, Th and U in granites and crustal protoliths; implications for the chemistry of crustal melts. J. Petrol. 1996, 7, 521-552. [CrossRef]

2. Förster, H.-J. The Variscan Granites of the Erzgebirge and Their Accessory Minerals. Ph.D. Thesis, Technical University Bergakademie Freiberg, Freiberg, Germany, 1998.

3. Förster, H.-J. The chemical composition of uraninite in Variscan granites of the Erzgebirge, Germany. Mineral. Mag. 1999, 63, 239-252. [CrossRef]

4. Trumbull, R.; Förster, H.-J.; Rhede, D. REE-Y-Th-U bearing accessory minerals and their contribution to the lanthanide and actinide trace-element budget in an anorogenic granite from the Erongo complex, NW Namibia. Z. Geol. Wiss. 2010, 38, 145-165.

5. Breiter, K. Monazite and zircon as major carriers of Th, $\mathrm{U}$, and $\mathrm{Y}$ in peraluminous granites: Examples from the Bohemian Massif. Mineral. Petrol. 2016, 110, 767-785. [CrossRef]

6. Förster, H.-J. The chemical composition of REE-Y-Th-U-rich accessory minerals in peraluminous granites of the ErzgebirgeFichtelgebirge region, Germany, Part I: The monazite-(Ce)-brabantite solid solution series. Am. Mineral. 1998, 83, $259-272$. [CrossRef]

7. Förster, H.-J. The chemical composition of REE-Y-Th-U-rich accessory minerals in peraluminous granites of the ErzgebirgeFichtelgebirge region, Germany, Part II: Xenotime. Am. Mineral. 1998, 83, 1302-1315. [CrossRef]

8. Förster, H.-J. Composition and origin of intermediate solid solutions in the system thorite-xenotime-zircon-coffinite. Lithos 2006, 88, 35-55. [CrossRef] 
9. Förster, H.-J.; Gottesmann, B.; Tischendorf, G.; Siebel, W.; Rhede, D.; Seltmann, R.; Wasternack, J. Permo-Carboniferous subvolcanic rhyolitic dikes in the western Erzgebirge/Vogtland, Germany: A record of source heterogeneity of post-collisional felsic magmatism. N. J. Miner. Abh. 2007, 183, 123-147. [CrossRef]

10. Breiter, K.; Čopjaková, R.; Škoda, R. The involvement of F, $\mathrm{CO}_{2^{-}}$, and As in the alteration of Zr-Th-REE-bearing accessory minerals in the Hora Svaté Kateřiny A-type granite, Czech Republic. Can. Mineral. 2009, 47, 1375-1398. [CrossRef]

11. Tischendorf, G. Silicic Magmatism and Metallogenesis of the Erzgebirge; Veröffentlichungen des Zentralinstituts für Physik der Erde: Potsdam, Germany, 1989; Volume 107, pp. 1-316.

12. Förster, H.-J.; Tischendorf, G.; Trumbull, R.B.; Gottesmann, B. Late-collisional granites in the Variscan Erzgebirge, Germany. J. Petrol. 1999, 40, 1613-1645. [CrossRef]

13. Förster, H.-J.; Romer, R.L. Carboniferous magmatism. In Pre-Mesozoic Geology of Saxo-Thuringia-From the Cadomian Active Margin to the Variscan Orogen; Linneman, U., Romer, R.L., Eds.; Schweizerbart: Stuttgart, Germany, 2010; pp. $287-308$.

14. Breiter, K. Nearly contemporaneous evolution of the A- and S-type fractionated granites in the Krušné hory/Erzgebirge Mts., Central Europe. Lithos 2012, 151, 105-121. [CrossRef]

15. Laube, G.C. Geologie des Böhmischen Erzgebirges Teil I; Commissions-Verlag von Fr. Řivnáč: Prag, Czech Republic, 1876; p. 238.

16. Teuscher, E.O. Primäre Bildungen des granitischen Magmas und seiner Restlösungen im Massif von Eibenstock-Neudeck. Miner. Petrogr. Mitt. 1936, 47, 211-262.

17. Breiter, K.; Sokolová, M.; Sokol, A. Geochemical specialization of the tin-bearing granitoid massifs of NW Bohemia. Miner. Depos. 1991, 26, 298-306. [CrossRef]

18. Tichomirowa, M.; Käßner, A.; Sperner, B.; Lapp, M.; Leonhardt, D.; Linnemann, U.; Münker, C.; Ovtcharova, M.; Pfänder, J.A.; Schaltegger, U.; et al. Dating multiply overprinted granites: The effect of protracted magmatism and fluid flow on dating sytems (zircon U-Pb: SHRIMP/SIMS, LA-ICP-MS, CA-ID-TIMS; and Rb-Sr, Ar-Ar) - Granites from the Western Erzgebirge (Bohemian Massif, Germany). Chem. Geol. 2019, 519, 11-38. [CrossRef]

19. Förster, H.-J.; Rhede, D. The Be-Ta-rich granite of Seiffen (eastern Erzgebirge, Germany): Accessory-mineral chemistry, composition and age of a late-Variscan Li-F granite of A-type affinity. N. J. Miner. Abh. 2006, 182, 307-321. [CrossRef]

20. Zhang, R.; Lehmann, B.; Seltmann, R.; Sun, W.; Li, C. Cassiterite U-Pb geochronology constrains magmatic-hydrothermal evolution in complex evolved granite systems: The classic Erzgebirge tin province (Saxony and Bohemia). Geology 2017, 45. [CrossRef]

21. Tomek, F.; Žák, J.; Svojtka, M.; Finger, F.; Waitzinger, M. Emplacement dynamics of syn-collapse ring dykes: An example from the Altenberg-Teplice caldera, Bohemian Massif. Bull. GSA 2019, 131, 997-1016. [CrossRef]

22. Breiter, K.; Frýda, J.; Seltmann, R.; Thomas, R. Mineralogical evidence for two magmatic stages in the evolution of an extremely fractionated P-rich rare-metal granite: The Podlesí stock. J. Petrol. 1997, 38, 1723-1739. [CrossRef]

23. Breiter, K.; Müller, A.; Leichmann, J.; Gabašová, A. Textural and chemical evolution of a fractionated granitic system: The Podlesí stock, Czech Republic. Lithos 2005, 80, 323-345. [CrossRef]

24. Whitney, D.L.; Evans, B.W. Abbreviations for names of rock-forming minerals. Am. Mineral. 2010, 95, 185-187. [CrossRef]

25. Linthout, $\mathrm{K}$. Tripartite division of the system $2 \mathrm{REEPO}_{4}-\mathrm{CaTh}\left(\mathrm{PO}_{4}\right)_{2}-2 \mathrm{ThSiO}_{4}$, discredition of brabantite, and recognition of cheralite as the name for members dominated by CaTh $\left(\mathrm{PO}_{4}\right)_{2}$. Can. Mineral. 2007, 45, 503-508. [CrossRef]

26. McDonough, W.F.; Sun, S. The composition of the Earth. Chem. Geol. 1995, 120, 223-253. [CrossRef]

27. Charoy, B.; Noronha, F. Multistage growth of a rare-element, volatile-rich microgranite at Argemela. J. Petrol. 1996, 37, 73-94. [CrossRef]

28. Raimbault, L.; Cuney, M.; Azencott, C.; Duthou, J.L.; Joron, J.L. Geochemical evidence for a multistage magmatic genesis of Ta-Sn-Li mineralization in the granite at Beauvoir, French Massif Central. Econ. Geol. 1995, 90, 548-596. [CrossRef]

29. Hösel, G. Das Zinnerz-Lagerstättengebiet Ehrenfriedersdorf/Erzgebirge. Bergbaumonographie 1994, 1, 189.

30. Huang, X.; Wang, R.C.; Chen, X.M.; Hu, H.; Liu, C.S. Vertical variation in the mineralogy of the Yichun topaz-lepidolite granite, Jiangxi province, Southern China. Can. Mineral. 2002, 40, 1047-1068. [CrossRef]

31. Kirkham, R.V.; Sinclair, W.D. Comb quartz layers in felsic intrusions and their relationship to porphyry deposits. Recent advances in the geology of granite-related deposits. Can. Inst. Min. Metall. 1988, 39, 50-71.

32. Breiter, K.; Śkoda, R.; Uher, P. Nb-Ta-Ti-W-Sn-oxide minerals as indicators of a peraluminous P- and F-rich granitic system evolution: Podlesí, Czech Republic. Mineral. Petrol. 2007, 91, 225-248. [CrossRef]

33. Mogilevsky, P. On the miscibility gap in monazite-xenotime systems. Chem. Geol. 2007, 34, 201-214. [CrossRef]

34. Breiter, K. Mineral and textural evaluation of subvolcanic A-type granite: Hora sv. Kateřiny stock, Krušné Hory Mts., Czech Republic. Z. Geol. Wiss. 2008, 36, 365-382.

35. Breiter, K.; Ďurišová, J.; Hrstka, T.; Korbelová, Z.; Hložková Vaňková, M.; Vašinová Galiová, M.; Kanický, V.; Rambousek, P.; Knésl, I.; Dobeš, P.; et al. Assessment of magmatic vs. metasomatic processes in rare-metal granites: A case study of the Cínovec/Zinnwald Sn-W-Li deposit, Central Europe. Lithos 2017, 292-293, 198-217. [CrossRef]

36. Seydoux-Guillaume, A.M.; Wirth, R.; Heinrich, W.; Montel, J.-M. Experimental determination of thorium partitioning between monazite and xenotime using analytical electron microscopy and X-ray diffraction Rietveld analysis. Eur. J. Mineral. 2002, 14, 869-878. [CrossRef]

37. Heinrich, W.; Andrehs, G.; Franz, G. Monazite-xenotime miscibility gap thermometry: I. An empirical calibration. J. Metamorph. Geol. 1997, 15, 3-17. [CrossRef] 
38. Lv, Z.-H.; Zhang, H.; Tang, Y. Lanthanide tetrads with implications for liquid immiscibility in an evolving magmatic-hydrothermal system: Evidence from rare earth elements in zircon from the No. 112 pegmatite, Kelumute, Chinese Altai. J. Asian Earth Sci. 2018, 164, 9-22. [CrossRef]

39. Liu, C.-Q.; Zhang, H. The lanthanide tetrad effect in apatite from the Altay No. 3 pegmatite, Xingjiang, China: An intrinsic feature of the pegmatite magma. Chem. Geol. 2005, 214, 61-77. [CrossRef]

40. Bau, M. Controls on the fractionation of isovalent trace elements in magmatic and aqueous systems: Evidence from $\mathrm{Y} / \mathrm{Ho}, \mathrm{Zr} / \mathrm{Hf}$, and lanthanide tetrad effect. Contrib. Mineral. Petrol. 1996, 123, 323-333. [CrossRef]

41. Irber, $\mathrm{W}$. The lanthanide tetrad effect and its correlation with $\mathrm{K} / \mathrm{Rb}, \mathrm{Eu} / \mathrm{Eu}^{*}, \mathrm{Sr} / \mathrm{Eu}, \mathrm{Y} / \mathrm{Ho}$, and $\mathrm{Zr} / \mathrm{Hf}$ of evolving peraluminous granite suites. Geochim. Cosmochim. Acta 1999, 63, 489-508. [CrossRef]

42. Monecke, M.; Dulski, P.; Kempe, U. Origin of convex tetrads in rare earth element patterns of hydrothermally altered siliceous igneous rocks from the Zinnwald Sn-W deposit, Germany. Geochim. Cosmochim. Acta 2007, 71, 335-353. [CrossRef]

43. Anenburg, M. Rare earth mineral diversity controlled by REE pattern shapes. Mineral. Mag. 2020, 84, 629-639. [CrossRef]

44. Duc-Tin, Q.; Keppler, H. Monazite and xenotime solubility in granitic melts and the origin of the lanthanide tetrad effect. Contrib. Mineral. Petrol. 2015, 169, 1-26. [CrossRef]

45. Anenburg, M.; Burnham, A.D.; Mavrogenes, J.A. REE redistribution textures in altered fluorapatite: Symplectites, veins and phosphate-silicate-carbonate assemblages from the Nolans Bore P-REE-Th deposit, NT, Australia. Can. Mineral. 2018, 56, 331-354. [CrossRef]

46. Hecht, L. Die Glimmer als Indikatoren für die magmatische und postmagmatische Entwicklung der Granite des Fichtelgebirges (NE Bayern). Münchner Geol. Hefte 1993, 10, 1-221.

47. Göd, R.; Oberlercher, G.; Brandstatter, F. Zur Geochemie und Mineralogie eines Monazit-führenden Granitkörpers im Südbohmischen Pluton (Gutau, Oberösterreich). J. Geol. Bundesanst. 1996, 139, 445-452.

48. Breiter, K. Short note on a thorium-rich granite in the Three Corner Area (Dreiländereck) of Austria, Czech Republic and Germany. J. Geol. Bundesanst. 2005, 145, 141-143.

49. Broska, I.; Williams, C.T.; Janák, M.; Nagy, G. Alteration and brealdown of xenotime-(Y) and monazite-(Ce) in granitic rocks of the Western Carpathians, Slovakia. Lithos 2005, 82, 71-83. [CrossRef]

50. Uher, P.; Plašienka, D.; Ondrejka, M.; Hraško, L.; Konečný, P. Uranium-rich monazite-(Ce) from the Krivá type granitic cobbles in conglomerates of the Pieniny Klippen Belt, Western Carpathians, Slovakia: Composition, age determination and possible source areas. Geol. Q. 2013, 57, 343-352.

51. Broska, I.; Kubiš, M. Accessory minerals and evolution of tin-bearing S-type granites in the western segment of the Gemeric Unit (Western Carpathians). Geol. Carpath. 2018, 69, 483-497. [CrossRef]

52. René, M. REE and Y mineralogy of the Krudum granite body (Saxothuringian zone). Minerals 2018, 8, 287. [CrossRef]

53. Förster, H.-J.; Rhede, D.; Hecht, L. Chemical composition of radioactive accessory minerals: Implications for the evolution, alteration, age, and uranium fertility of the Fichtelgebirge granites (NE Bavaria, Germany). N. J. Miner. Abh. 2008, 185, 161-182. [CrossRef]

54. Finger, F.; Broska, I.; Roberts, M.P.; Schermaier, A. Rerplacement of primary monazite by apatite-allanite-epidote coronas in an amphibolite facies granite gneiss from the eastern Alps. Am. Mineral. 1998, 83, 248-258. [CrossRef]

55. Pérez-Soba, C.; Villaseca, C.; Orejana, D.; Jeffries, F. Uranium-rich accessory minerals in the peraluminous and perphosphorous Belvís de Monroy pluton (Iberian Variscan belt). Contrib. Mineral. Petrol. 2014, 167, 1008. [CrossRef]

56. Hoshino, M.; Watanabe, Y.; Ishihara, S. Crystal chemistry of monazite from the granitic rocks of Japan: Petrogenetic implications. Can. Mineral. 2012, 50, 1331-1346. [CrossRef] 